\title{
Upper-Tropospheric Environment-Tropical Cyclone Interactions over the Western North Pacific: A Statistical Study
}

\author{
Yu-Kun QIAN ${ }^{* 1,2}$, Chang-Xia LIANG ${ }^{3}$, Zhuojian YUAN ${ }^{1}$, Shiqiu PENG ${ }^{2}$, Junjie WU $^{4}$, and Sihua WANG ${ }^{5}$ \\ ${ }^{1}$ Center for Monsoon and Environmental Research/Department of Atmospheric Science, Sun Yat-sen University, Guangzhou 510275 \\ ${ }^{2}$ State Key Laboratory of Tropical Oceanography, South China Sea Institute of Oceanology, \\ Chinese Academy of Sciences, Guangzhou 510301 \\ ${ }^{3}$ South China Sea Marine Prediction Center, South Oceanic Administration, Guangzhou 510300 \\ ${ }^{4}$ Civil Aviation Flight University of China, Guanghan 618307 \\ ${ }^{5}$ Luogang District Meteorological Bureau, Guangzhou 510530
}

(Received 13 June 2015; revised 16 September 2015; accepted 22 October 2015)

\begin{abstract}
Based on 25-year (1987-2011) tropical cyclone (TC) best track data, a statistical study was carried out to investigate the basic features of upper-tropospheric TC-environment interactions over the western North Pacific. Interaction was defined as the absolute value of eddy momentum flux convergence (EFC) exceeding $10 \mathrm{~m} \mathrm{~s}^{-1} \mathrm{~d}^{-1}$. Based on this definition, it was found that $18 \%$ of all six-hourly TC samples experienced interaction. Extreme interaction cases showed that EFC can reach $\sim 120 \mathrm{~m} \mathrm{~s}^{-1} \mathrm{~d}^{-1}$ during the extratropical-cyclone (EC) stage, an order of magnitude larger than reported in previous studies. Composite analysis showed that positive interactions are characterized by a double-jet flow pattern, rather than the traditional trough pattern, because it is the jets that bring in large EFC from the upper-level environment to the TC center. The role of the outflow jet is also enhanced by relatively low inertial stability, as compared to the inflow jet. Among several environmental factors, it was found that extremely large EFC is usually accompanied by high inertial stability, low SST and strong vertical wind shear (VWS). Thus, the positive effect of EFC is cancelled by their negative effects. Only those samples during the EC stage, whose intensities were less dependent on VWS and the underlying SST, could survive in extremely large EFC environments, or even re-intensify. For classical TCs (not in the EC stage), it was found that environments with a moderate EFC value generally below $\sim 25 \mathrm{~m} \mathrm{~s}^{-1} \mathrm{~d}^{-1}$ are more favorable for a TC's intensification than those with extremely large EFC.
\end{abstract}

Key words: Tropical cyclone-trough interaction, upper troposphere, eddy momentum flux convergence

Citation: Qian, Y.-K., C.-X. Liang, Z. J. Yuan, S. Q. Peng, J. J. Wu, and S. H. Wang, 2016: Upper-tropospheric environmenttropical cyclone interactions over the western North Pacific: A statistical study. Adv. Atmos. Sci., 33(5), 614-631, doi: 10.1007/s00376-015-5148-x.

\section{Introduction}

Track and intensity forecasts are two major concerns in tropical cyclone (TC) research. Particular emphasis has been placed on the former of these two aspects, leading to a substantial increase in the skill of TC track prediction. Meanwhile, TC intensity forecasts have received virtually no improvement (e.g., Emanuel, 2000). This lagging behind of intensity forecast skill, which is a widely accepted fact (e.g., Elsberry et al., 1992; Fitzpatrick, 1997; DeMaria et al., 2007; Montgomery and Smith, 2014; Smith and Montgomery, 2015), means that greater effort should be put into researching TC intensity.

\footnotetext{
* Corresponding author: Yu-Kun QIAN

Email: qianyk@mail3.sysu.edu.cn
}

It has long been known that upper-tropospheric environmental flow is an important factor that could modulate the intensity change of a TC. Two reasons are proposed, based on Sawyer-Eliassen balance (SEB) vortex theory (Eliassen, 1952; Sawyer, 1956). The first is that, in the upper troposphere, a TC does not retain its axisymmetric structure and thus environmental asymmetric forcing is generally stronger than that of the middle or lower troposphere (e.g., Pfeffer and Challa, 1981). The second is that the inertial stability in the upper layer, representing the resistance of the vortex axisymmetric response to environmental forcings, is much weaker than that in the middle or lower layers (e.g., Holland and Merrill, 1984). Therefore, upper-level asymmetric forcings will excite larger vortex responses, which could easily penetrate into the core region and thus result in TC's intensity change. This phenomenon is also termed as upper-level environment- 


\section{TC interaction.}

A large number of interaction cases have been reported in the literature, such as Hurricanes (or Tropical Storms) Dorothy (1966) (Erickson, 1967), Elena (1985) (Molinari and Vollaro, 1989), Danny (1985) (Molinari et al., 1998), Florence (1988) (Rodgers et al., 1991; Shi et al., 1997), Opal (1995) (Rodgers et al., 1998; Bosart et al., 2000), Bertha (1996) (Hanley, 2002) and Gabrielle (2001) (Molinari et al., 2006), over the Atlantic basin; Typhoons (or Tropical Storms) Flo (1990) (Wu and Cheng, 1999), Gene (1990) (Wu and Cheng, 1999), Winnie (1997) (Li et al., 2006), Olga (1999) (Yu and Kwon, 2005), Prapiroon (2000) (Yu and Kwon, 2005) and Haima (2004) (Qian et al., 2011), over the western North Pacific basin; as well as Tropical Cyclone Dora (2007) (Leroux et al., 2013) over the southwest Indian Ocean. These cases also show that intensification induced by upper-level environments can be found in all stages of TCs, such as tropical depression formation (Bracken and Bosart, 2000), tropical depression (Bosart and Bartlo, 1991) or tropical storm (Shi et al., 1997; Molinari et al., 1998) to hurricane transition, rapid intensification to a category 5 hurricane (Bosart et al., 2000) or supertyphoon (Titley and Elsberry, 2000), and tropical storm (Qian et al., 2011) or typhoon (Li et al., 2006) to extratropical cyclone transition.

Early case studies (e.g., Erickson, 1967) identified interaction visually from weather maps, by observing an upperlevel westerly trough approaching a TC. Thus, such interaction is also termed TC-trough interaction (e.g., Kimball and Evans, 2002; Yu and Kwon, 2005). Later, a typical case, Hurricane Elena (1985), was studied at length by Molinari and colleagues (Molinari and Vollaro, 1989, 1990; Molinari et al., 1995). In this case, they adopted the SEB vortex model to diagnose the relationship between eddy fluxes and Elena's intensity change and found that $200-\mathrm{hPa}$ eddy angular momentum flux convergence (EFC) significantly increased as an upper-level trough swept over Elena's northern part. From then on, the EFC value, which can be computed quantitatively and objectively, has been commonly used as a diagnostic for identifying TC-trough interaction, although the role of EFC in TC intensification was also explored in pioneering works (e.g., Sundqvist, 1970; Challa and Pfeffer, 1980; Pfeffer and Challa, 1981) that used idealized models.

Environmental EFC larger than a certain value is usually adopted to identify whether interaction has occurred (e.g., Molinari et al., 2006; Chen et al., 2015). Although many of the above cases underwent intensification when they interacted with upper-level flow using the EFC criterion, there were also weakened or decayed cases (e.g., Lewis and Jorgensen, 1978). Therefore, interaction with upper-level flows does not guarantee TC intensification. In order to verify the effect of upper-level flow on TC intensity, statistical studies are also carried out. Merrill (1988a, b) systematically investigated a large sample of observed outflow-layer wind data near Atlantic hurricanes. The common features of the upper-tropospheric flow pattern for intensifying and nonintensifying hurricanes were summarized, using a rotatedcoordinate composite technique rather than the EFC diagnos- tic. DeMaria et al. (1993) investigated the relation between intensity change and $200-\mathrm{hPa}$ EFC with 3 -yr records of Atlantic hurricanes. They found that about $1 / 3$ of the TCs intensified just after the enhanced EFC. The reason for TCs not intensifying was mostly increased vertical wind shear (VWS). Hanley et al. (2001) also examined TC-trough interaction, but using 12-yr records of Atlantic hurricanes and the composite technique. After excluding records that were over cold water or close to land, they separated the interaction events into four composites. Their results showed that $78 \%$ of superposition and $61 \%$ of distant interaction cases deepened. Although these results confirm that interaction favors TC intensification in a statistical sense, to identify whether or not a particular trough interaction is conducive to TC intensification is still not straightforward (Hanley et al., 2001; Leroux et al., 2013). Besides, these two studies ignored the role of upper-level inertial stability, emphasized in a number of other studies (e.g., Holland and Merrill, 1984; Rappin et al., 2011).

Since the comprehensive work of Hoskins et al. (1985), the perspective of isentropic potential vorticity (PV) has been introduced for viewing dynamical processes in the atmosphere. The PV perspective is not only applicable to TC motion (e.g., Wu and Wang, 2000; Chan et al., 2002; Choi et al., 2013), but also to TC intensity change (e.g., Molinari et al., 1998). Molinari et al. (1998, 1995) also applied the PV perspective to interaction cases, in which interaction could be viewed as an upper-level synoptic PV anomaly (the environment) interacting with a lower-level mesoscale PV anomaly (the TC). From such a perspective, although interaction is clearly and effectively shown as a superposition of two PV anomalies, there is no quantitative way of identifying whether interaction has occurred. Therefore, the EFC diagnostic is more frequently used than the PV perspective.

To date, statistical studies in this field have been relatively less common-especially for the western North Pacific, where TCs are most active compared with other basins. Therefore, the climatology of TC-upper-level-flow interactions and its possible impact on TC intensity over this basin remain unclear. Before addressing the "good trough/bad trough" issue, we should first carry out a preliminary but thorough study to reveal more basic features of upperlevel environment-TC interactions, especially for the western North Pacific basin. For example: What are the characteristics of upper-level flow patterns during interactions, besides the well-known trough signature? What is the relationship between the upper-level environmental factor and other largescale factors (e.g., SST and VWS) in controlling TC intensity over this basin? What is the nature of inertial stability during interactions? The present paper aims to address these aspects by examining 25 -yr TC records and revealing statistical characteristics of upper-tropospheric environment-TC interaction over the western North Pacific.

Section 2 describes the data and method. Section 3 presents the climatology of interactions. Section 4 describes the characteristics of upper-level flow patterns. The relationship between environmental factors and TC intensity change follows in section 5, and conclusions are given in section 6 . 


\section{Data and method}

\subsection{Data description}

The TC "best track" data over the western North Pacific were obtained from the Regional Specialized Meteorological Centre (RSMC), Tokyo. Given that aircraft reconnaissance of TCs in this basin terminated in 1987, making observations of TCs thereafter completely dependent on satellite retrieval (Martin and Gray, 1993), for consistency, only data after 1987 were used. Specifically, 25 years of data, from 1987 to 2011, including six-hourly TC positions, near-center maximum surface wind speed, and minimum sea level pressure, were chosen for the present study.

ERA-Interim (Dee et al., 2011) data were employed to describe the TC environment. The benefits of using these reanalysis data for studying the upper-level environment of TCs have been demonstrated in a number of studies (Molinari and Vollaro, 1990; Molinari et al., 1992). In the present study, six-hourly pressure-level wind fields, as well as SST data, on a $1.5^{\circ} \times 1.5^{\circ}$ grid, were used.

\subsection{Method}

In order to identify upper-level environment-TC interactions, EFC was computed in storm-relative cylindrical coordinates. The cylindrical coordinates contained 36 grids in the azimuthal direction, with $10^{\circ}$ intervals and 28 grids in the radial direction with $0.3^{\circ}$ intervals (about $33.3 \mathrm{~km}$ ). Latitude/longitude gridded data were interpolated to the cylindrical gridded data using a 16-neighbouring-point bicubic polynomial. EFC was calculated following Molinari and Vollaro (1990):

$$
\mathrm{EFC}=-\frac{1}{r^{2}} \frac{\partial}{\partial r} r^{2} \overline{u^{\prime} v^{\prime}}
$$

where $v$ and $u$ are the tangential and radial components of the storm-relative wind vector (i.e., after subtracting the translation velocity of a TC from the full wind vector), $r$ is the distance to the TC center, the overbar is the azimuthal mean, and the prime is the deviation from the mean. Consistent with previous studies (e.g., Hanley et al., 2001), the units of measurement for EFC is $\mathrm{m} \mathrm{s}^{-1} \mathrm{~d}^{-1}$. Molinari and Vollaro (1989) showed that the errors of EFC calculated in the inner radii (within $300 \mathrm{~km}$ ) may exceed $40 \%$. EFC evaluated at large radii is more reliable; however, it may not have an immediate impact on TC intensity. Therefore, the present study used a radial average from 300 to $600 \mathrm{~km}$ to identify TC-upper-flow interactions, following Hanley et al. (2001).

According to the SEB theory, within a slowly evolving TC, the TC's axisymmetric secondary circulation is largely balanced by several forcings, including EFC. This implies that if other forcings are not important, larger EFC will result in a stronger response of the TC's secondary circulation. If the strength of the TC's secondary circulation is positively correlated with its intensity, which is generally true, then one may expect a simple rule that larger EFC is more likely to enhance the outflow and thus intensify the TC (e.g., Challa and Pfeffer, 1980; Pfeffer and Challa, 1981; Molinari and Vollaro,
1990; Qian et al., 2011). However, when EFC becomes large, other factors may come into play.

One of the important factors concerning a TC's interaction with upper-level environmental flow, besides EFC, is the inertial stability, usually defined in the SEB theory (e.g., Rappin et al., 2011) as

$$
I=\overline{\zeta_{\mathrm{a}}}\left(f+\frac{2 \bar{v}}{r}\right),
$$

in which the azimuthal-averaged absolute vorticity $\overline{\zeta_{\mathrm{a}}}$ is defined as

$$
\overline{\zeta_{\mathrm{a}}}=\bar{f}+\bar{\zeta}=\bar{f}+\frac{\partial \bar{v}}{\partial r}+\frac{\bar{v}}{r}
$$

where $\zeta$ is relative vorticity and $f$ is planetary vorticity. Notice that the factor of $(f+2 \bar{v} / r)$ also appears in the momentum forcing functions [e.g., Molinari and Vollaro, 1990; see the first term on the rhs of their Eq. (2)]. When simultaneously taking into account the forcing effect of momentum source and the resistance effect of inertial stability, the factor of $(f+2 \bar{v} / r)$ can be eliminated. Therefore, the axisymmetric outflow is proportional to a forcing function in terms of EFC [rather than EFC multiplied by $(f+2 \bar{v} / r)$ ] and inversely proportional to the inertial stability, proxied by $\overline{\zeta_{\mathrm{a}}}$ (rather than I). In the SEB theory, it is generally required that the azimuthal-averaged absolute vorticity $\bar{\zeta}_{\mathrm{a}}>0$ (inertial stable). Smaller $\overline{\zeta_{\mathrm{a}}}$ indicates less resistance to the environmental forcing (e.g., EFC) and a larger radial extent of the vortex response (e.g., Holland and Merrill, 1984). In the present study, the absolute vorticity $\zeta_{\mathrm{a}}$ before azimuthal averaging was used to represent localized inertial stability:

$$
\zeta_{\mathrm{a}}=f+\zeta=f+\left(\frac{1}{r} \frac{\partial v r}{\partial r}-\frac{1}{r} \frac{\partial u}{\partial \lambda}\right)
$$

where $\lambda$ is the azimuth.

Besides EFC and inertial stability, two other environmental factors, SST and VWS, were also computed in cylindrical coordinates for each six-hourly TC record. According to Molinari and Vollaro (1993), VWS is defined as the difference between the 200- and $850-\mathrm{hPa}$ wind vector averaged within $500 \mathrm{~km}$ from the TC's center:

$$
\mathrm{VWS}=\sqrt{\left[\langle u\rangle_{200}-\langle u\rangle_{850}\right]^{2}+\left[\langle v\rangle_{200}-\langle v\rangle_{850}\right]^{2}} .
$$

In this definition, the angled brackets indicate area-weighted averaging:

$$
\langle A\rangle=\frac{1}{\sum_{j=J_{0}}^{j=J} \sin \beta_{j}} \sum_{j=J_{0}}^{j=J} \overline{A_{j}} \sin \beta_{j},
$$

where $\beta_{j}=0.3 j$ is the radial angle, in degrees (interval of $\sim 33.3 \mathrm{~km}$ ), and $j$ is the radial grid index, starting from $J_{0}$ to $J$. $J_{0}=0$ and $J=15$ are used for averaging within $500 \mathrm{~km}$. Similarly, SST is also averaged within $500 \mathrm{~km}$. 


\section{Climatology of upper-tropospheric TC- environment interaction}

\subsection{Characteristics of the dataset}

The "best track" dataset from the RSMC used in the resent study contains 628 TCs. Records at 0000, 0600, 1200 and 1800 UTC were chosen, yielding 21685 six-hourly samples, including tropical depressions (TD), tropical storms (TS), typhoons (TY), and extratropical cyclones (EC). Since the interaction could occur at any stage of a TC (Hanley et al., 2001), keeping all these records yields more samples for reliable statistics. TC intensity change is defined as the forward difference of sea-level minimum central pressure (SLP) i.e., $\Delta p(t)=p(t+\Delta t)-p(t)$ where $\Delta t=6 \mathrm{~h}$. Negative (positive) pressure change means intensification (weakening), and zero means no change.

Firstly, the 200-hPa EFC averaged within the 300-600 $\mathrm{km}$ radial band for all 21685 samples was calculated. Figure 1 shows the distribution of the results. Following previous studies (e.g., DeMaria et al., 1993; Hanley et al., 2001), an EFC value of $10 \mathrm{~m} \mathrm{~s}^{-1} \mathrm{~d}^{-1}$ is usually defined for identifying interactions. In the present study, interaction is defined similarly, but an EFC value above $10 \mathrm{~m} \mathrm{~s}^{-1} \mathrm{~d}^{-1}$ means positive interaction, while EFC below $-10 \mathrm{~m} \mathrm{~s}^{-1} \mathrm{~d}^{-1}$ means negative interaction. According to this definition, about $17.7 \%$ of the samples experience interaction: $3.7 \%$ negative and $14.0 \%$ positive. The proportion of positive interaction (14.0\%) is smaller than that $(\sim 23 \%)$ over the Atlantic basin, as shown by Hanley et al. (2001). More positive than negative samples over the western North Pacific basin, which is quite similar to the situation over the Atlantic basin, indicates that a positive EFC environment is more favorable for the maintenance of TCs than a negative one. However, a larger EFC value does not guarantee a greater number (or larger proportion) of intensifying samples, as can be seen from Fig. 1.

Figure 2 shows the percentage of different TC types, including TDs, TSs, TYs and ECs, as identified by the RSMC. The TS and TY samples account for $37 \%$ and $25 \%$ of the total, respectively, resulting in $62 \%$ of samples being canonical tropical cyclones (i.e., TSs and TYs). Besides, $26 \%$ of samples are TDs and $12 \%$ are ECs. Each sample type has a proportion that interacts with upper-level flows. This result verifies that interaction can occur at any stage of development. We can also see that, although the proportion of EC samples is least, almost $60 \%$ of them experience interaction with upper-level flows. This ratio is significantly larger than for the other three types, showing that EC samples are more likely to interact with upper-level flow, while it is relatively rare to see interaction in the other three types.

\subsection{Spatial distribution}

The spatial distribution of interaction samples over the western North Pacific is shown in Fig. 3a. The southwest to northeast orientation of the distribution from the South China Sea to the Bering Sea suggests that interactions generally occur after a TC's recurvature to the northeast. The maximum frequency of occurrence of 0.5 per year per grid can be found around Japan. The interaction active regions are within $\left(25^{\circ}-\right.$ $\left.45^{\circ} \mathrm{N}, 125^{\circ}-165^{\circ} \mathrm{E}\right)$, with two peaks at about $30^{\circ} \mathrm{N}$ and $40^{\circ} \mathrm{N}$ (Fig. 3b), and $138^{\circ} \mathrm{E}$ and $151^{\circ} \mathrm{E}$ (Fig. 3c), respectively. These results indicate that most interactions occur when TCs move into the midlatitudes, right under the influence of upper-level westerlies, but before dissipating. Notice that there are still many interaction samples (434) south of $20^{\circ} \mathrm{N}$, over both the north South China Sea and east of the Philippines. More than $60 \%$ (276) are during the genesis or development stages (before reaching their peak intensities) when interaction occurs.

\section{Characteristics of upper-level flow patterns during interaction}

Previous studies (e.g., Hanley et al., 2001) have already identified the most common upper-level flow, i.e., a westerly trough pattern during interaction. Therefore, the interaction is also named TC-trough interaction. However, EFC is an abstract index and EFC larger than a certain threshold does not necessarily mean the flow would be characterized by a westerly trough. There could be other types of flow patterns with different synoptic signatures, although they may be related more or less to a "V"-shaped trough in the vicinity of a TC. Summarizing these features of the flow pattern will help to identify interactions using upper-level wind fields only.

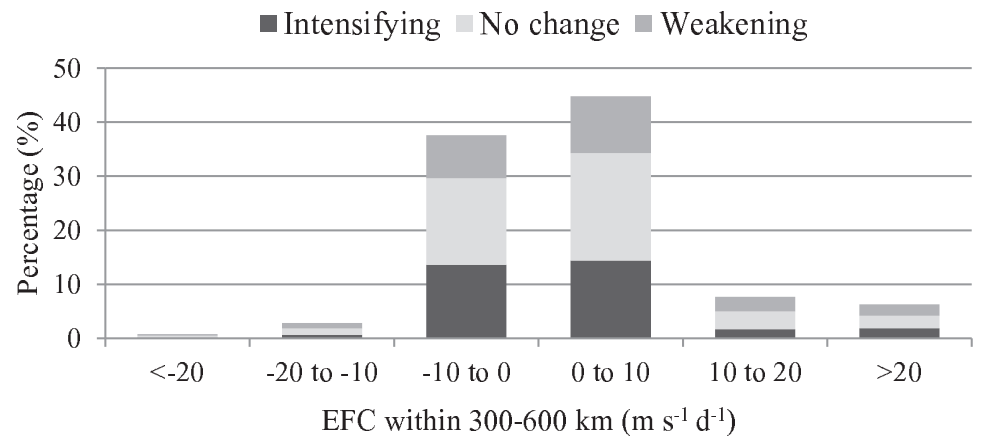

Fig. 1. Distribution of 200-hPa EFC averaged over a 300-600 km radius. There were 21685 samples in total from 1987 to 2011. 


\section{all samples $\quad$ interaction samples}

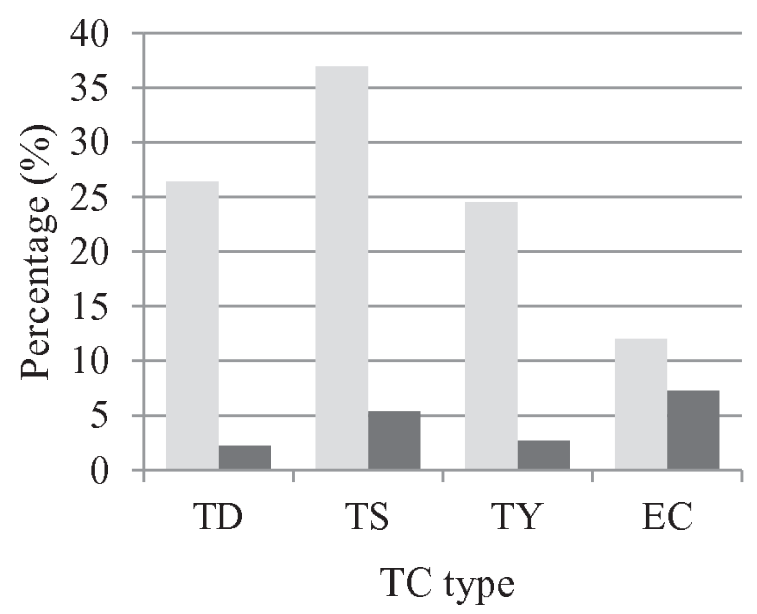

Fig. 2. Distributions of different TC types. There were 21685 samples in total and 3890 interaction samples from 1987 to 2011.

\subsection{Extreme interaction}

Firstly, extreme interactions (extremely large magnitudes of EFC) from 1987 to 2011 were identified. Table 1 details the basic information of three negative and three positive extreme interaction samples. The three negative samples have EFC values of about $-70 \mathrm{~m} \mathrm{~s}^{-1} \mathrm{~d}^{-1}$. The three positive interactions show EFC values larger than $120 \mathrm{~m} \mathrm{~s}^{-1} \mathrm{~d}^{-1}$, almost twice that of the negative interactions in magnitude. The identified extreme positive EFC values are much larger than those reported previously in the literature [e.g., $\sim 40 \mathrm{~m} \mathrm{~s}^{-1}$ $\mathrm{d}^{-1}$, as shown by $\mathrm{Wu}$ and Cheng (1999)]. Except for TC Andy (1989), which was in the TS stage, the other five samples were all in the EC stage. Low SST and large VWS are their common environmental features. It is noticeable that the negative extreme interaction samples could undergo filling $(\Delta p>0)$, while the positive ones could intensify further. Since these interaction samples were mainly in the EC stage, their intensity (i.e., minimum SLP) may not have depended as strongly on SST and VWS as it would in the TS or TY stages, for example. Extremely large positive EFC from upper-level flows would possibly have been responsible for their intensification.

Figure 4 shows the 200-hPa flows for three extreme negative interaction samples (Figs. $4 \mathrm{a}-\mathrm{c}$ ) and three positive samples (Figs. 4d-f). All six samples show that extreme EFC is associated with upper-level westerly troughs. For extreme negative interaction, TCs are right under the influence of northwesterly wind upstream of the trough axes and tend to (a)

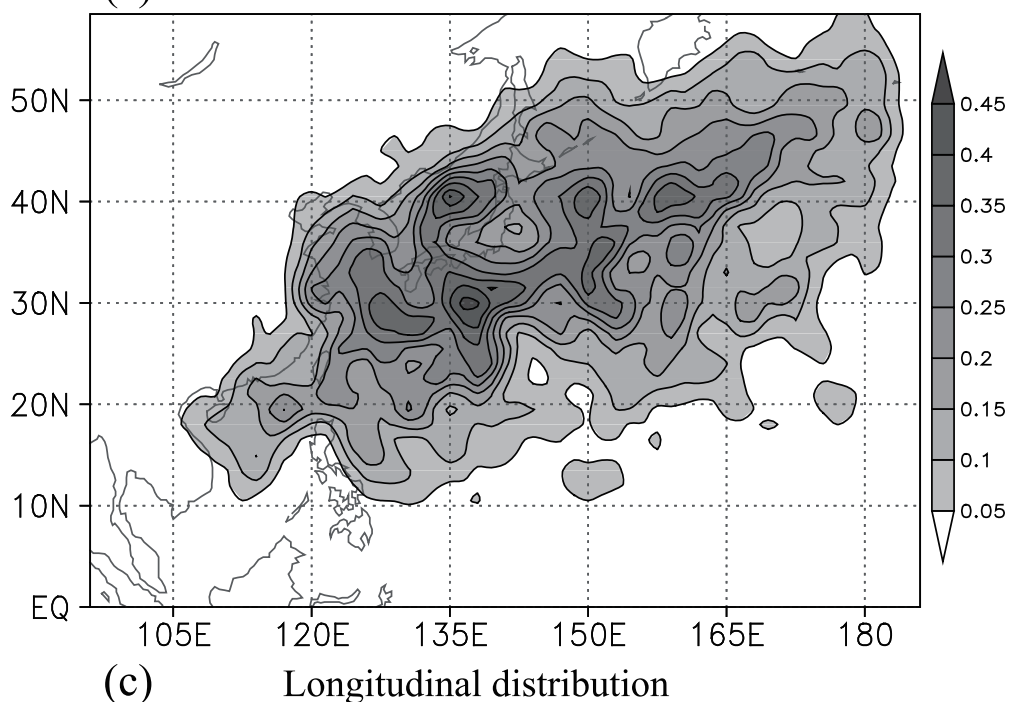

(b)Latitudinal distribution

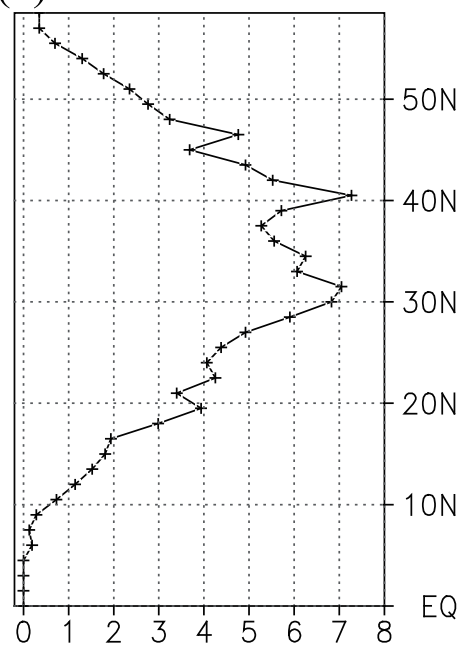

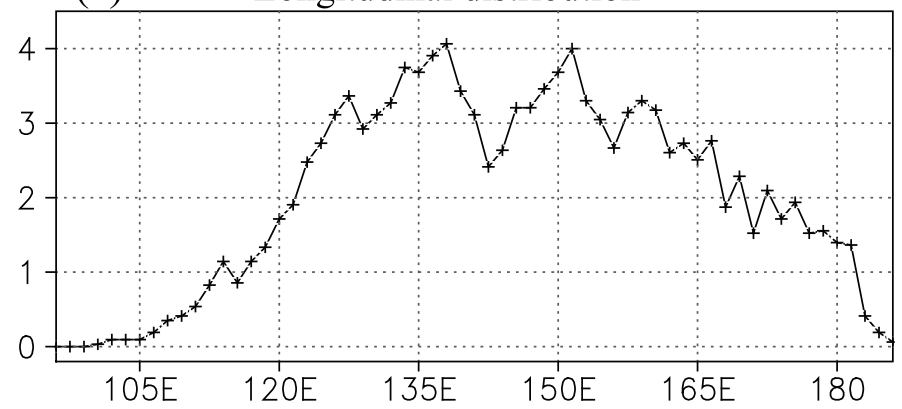

Fig. 3. (a) Spatial (interval: 0.05 per square grid per year; 9-point smoothing operator applied), (b) latitudinal and (c) longitudinal distributions of the interaction samples from 1987 to 2011 . The plots are on a $1.5^{\circ} \times 0.5^{\circ}$ grid. 
Table 1. TC samples of extreme interactions. Environmental SST and VWS are averaged within $500 \mathrm{~km}$ and EFC is averaged within the $300-600 \mathrm{~km}$ radial band.

\begin{tabular}{|c|c|c|c|c|c|c|c|c|c|}
\hline & Name & Type & Time (UTC) & $\operatorname{SST}\left({ }^{\circ} \mathrm{C}\right)$ & $\operatorname{VWS}\left(\mathrm{m} \mathrm{s}^{-1}\right)$ & $\operatorname{EFC}\left(\mathrm{m} \mathrm{s}^{-1} \mathrm{~d}^{-1}\right)$ & $\overline{\zeta_{\mathrm{a}}}\left(10^{-5} \mathrm{~s}^{-1}\right)$ & $\operatorname{Pmin}(\mathrm{hPa})$ & $\Delta p(\mathrm{hPa})$ \\
\hline \multirow[t]{3}{*}{ Negative extreme } & Andy & TS & 120024 Apr 1989 & 23.3 & 29.3 & -73.6 & 6.5 & 992 & 2 \\
\hline & Ewiniar & $\mathrm{EC}$ & 180020 Aug 2000 & 21.1 & 26.9 & -66.9 & 8.7 & 1004 & 4 \\
\hline & Nalgae & $\mathrm{EC}$ & 060027 Jul 2005 & 15.4 & 28.4 & -70.1 & 10.8 & 1006 & 0 \\
\hline \multirow[t]{3}{*}{ Positive extreme } & Nelson & $\mathrm{EC}$ & 060010 Oct 1988 & 12.7 & 43.6 & 121.0 & 8.9 & 980 & -8 \\
\hline & Forrest & $\mathrm{EC}$ & 180029 Oct 1989 & 11.6 & 34.1 & 116.7 & 12.7 & 956 & -4 \\
\hline & Shanshan & $\mathrm{EC}$ & 180024 Sep 2000 & 14.5 & 14.1 & 133.9 & 12.0 & 956 & 0 \\
\hline
\end{tabular}

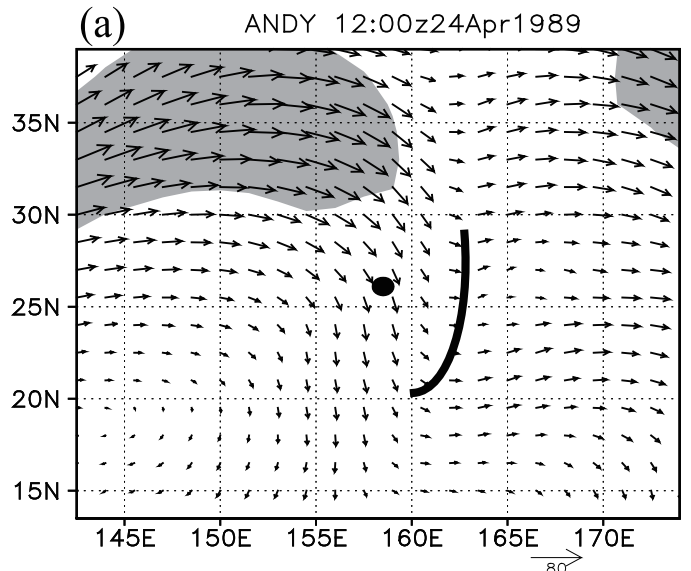

(b)

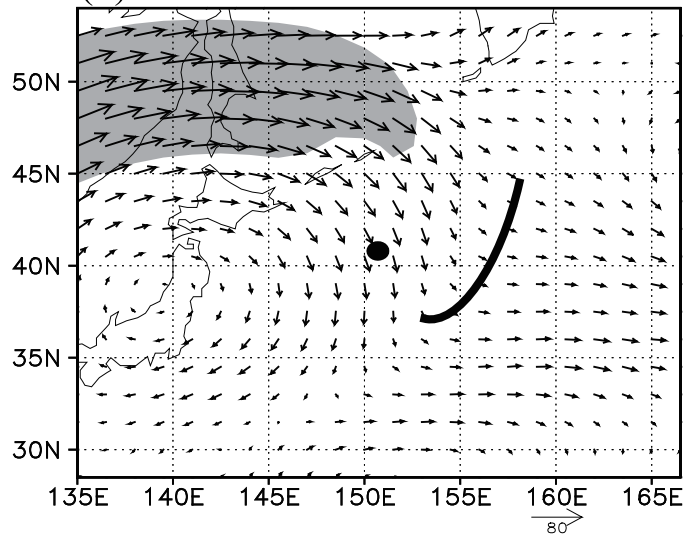

(c)

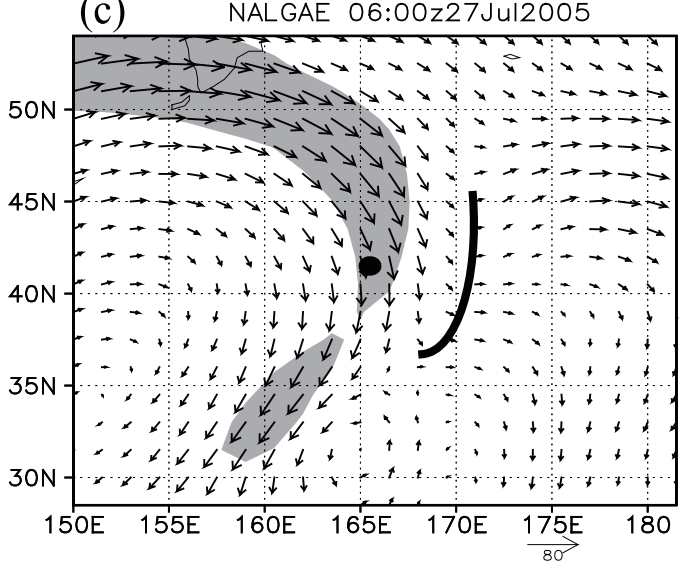

(d) NELSON 06:00z100ct1988

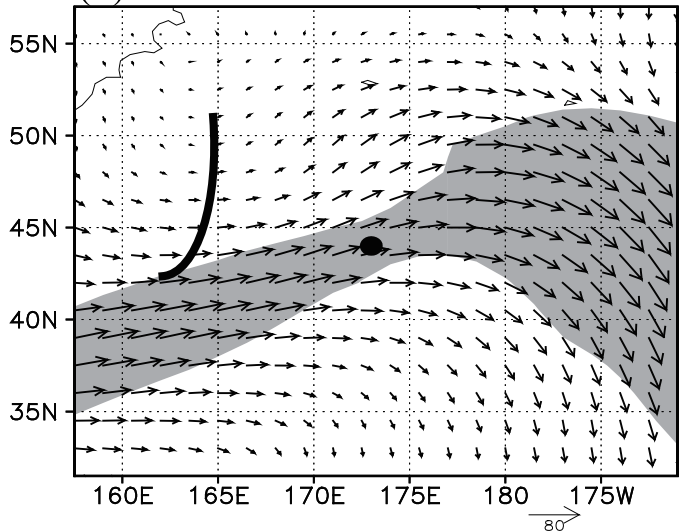

(e) FORREST 18:00z290ct1989

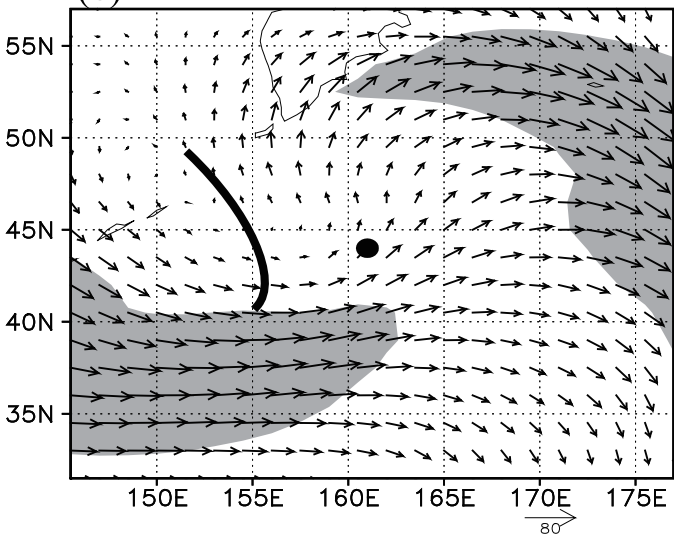

(f) SHANSHAN 18:00z24Sep2000

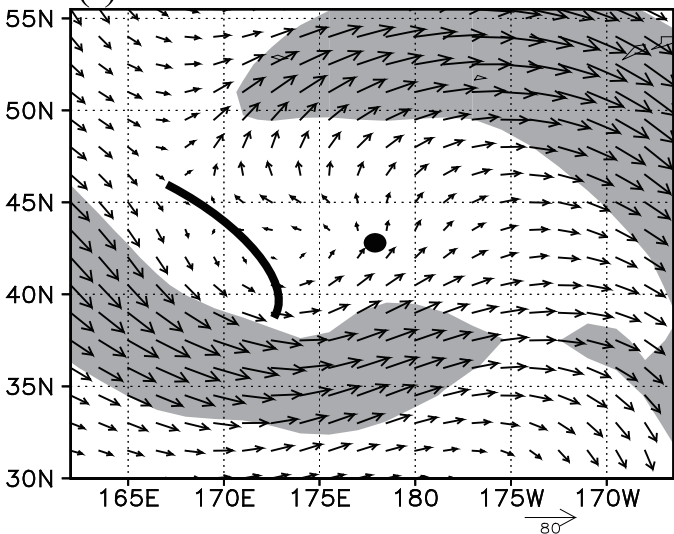

Fig. 4. 200-hPa wind fields $\left(\mathrm{m} \mathrm{s}^{-1}\right.$; shaded for wind speed $>50 \mathrm{~m} \mathrm{~s}^{-1}$ ) for $(\mathrm{a}-\mathrm{c})$ three TC samples experiencing extreme negative EFC values and (d-f) three TC samples experiencing extreme positive EFC values. Black dots show TC locations; thick solid lines indicate upper-layer troughs. 
dissipate. In contrast, the three positive interactions cases (Figs. 4d-f) were under the influence of southwesterly wind ahead of the troughs axis and retained a relatively high level of intensity compared to the negative cases (Table 1). Experiencing extremely large EFC, these TC samples were mostly close to westerly jet streams (shaded areas in Fig. 4), or even inside the jet core regions. Although strong wind speed associated with the jets exerted large asymmetric forcing that resulted in large EFC, it also brought strong VWS to the TCs (Table 1). In such a strongly sheared environment, TCs cannot maintain their typical structure and usually undergo transition into ECs.

\subsection{All positive interaction}

According to the SEB theory, positive (negative) interaction would transport cyclonic (anticyclonic) angular momentum from the upper-level environment to TCs and enhance (reduce) their outflows. Due to its possible effects on TC intensification, previous studies have tended to concentrate on positive interaction. Following this line, we focused on upper-level flow patterns of all positive interaction samples. To extract the characteristics of the flow pattern from a large number of samples, the composite technique was used. Although the composite may smooth out some important characteristics of individual TCs, it emphasizes the signatures that repeatedly appear.

There were several considerations regarding the composite procedure. Firstly, as EFC is computed using stormrelative wind (i.e., the translation speed of the TC is subtracted), it would be more meaningful to examine the stormrelative wind pattern. Secondly, to reduce the smoothing effect of the composite technique and to obtain more flow patterns, the samples were divided into eight groups according to the storm-relative wind directions averaged within $500 \mathrm{~km}$ of TCs. It is worth noting that different synoptic patterns or different positions of troughs will be somehow smoothed in the composite. However, there is no best way to group individual samples into different flow patterns, and different composite criteria would also suffer from the same problem. The present choice was shown to be effective in the sense of keeping flow features in the regions near the TC center and minimizing the blurring effect not too far from TCs. Besides, the most domi- nant flow type, the trough pattern, should be most frequently identified, in accordance with previous studies. Thirdly, different TCs may locate at different latitudes. Thus, traditional composite analysis using the latitude-longitude gridded wind field at different latitudes may be compromised by the problem of the meridian converging at high latitudes, and thus blurring some flow features. Taking this into account, all the composites were performed in cylindrical coordinates on a sphere with respect to their origins (i.e., the TCs' centers).

Table 2 lists the statistics of the eight composites and Fig. 5 shows the corresponding composite flow patterns as well as localized EFC. Local EFC is defined similar to Eq. (1), except that no azimuthal average is taken so that the local EFC is also a function of azimuth. Not surprisingly, the southwest composite contains 1359 samples, which is almost half of all positive interaction samples (Table 2), indicating that classical positive interactions usually occur right under the southwesterly wind between a westerly trough and a ridge (Fig. 5h). Two westerly jets (black bold arrows) locate southwest and northeast of the TC's center, respectively, and the TC's axisymmetric outflow tends to enhance the northeast outflow jet but weaken the southwest inflow one. The southwest jet imports cyclonic eddy angular momentum to the TC, while the northeast jet exports anticyclonic eddy angular momentum from the TC. Both jets result in positive EFC channels (red areas), so that the 300-600 $\mathrm{km}$ radial band has the largest mean EFC value (26.9 $\mathrm{m} \mathrm{s}^{-1} \mathrm{~d}^{-1}$ ) among the eight composites. The west composite (Fig. 5a), containing 983 samples, shows a strong westerly jet crossing the TC's center. A smaller amplitude of the synoptic disturbance also indicates a smaller curvature of the flow and larger wind speeds, as compared to the southwest composite. The south composite has 323 samples, which is the third highest among the eight composites. The corresponding flow pattern (Fig. $5 \mathrm{~g}$ ) is similar to that of the southwest composite, except that the trough to the west of the TC center intensifies with its bottom extending to the south of the TC. The wind near the TC center is a uniform southerly, but weaker compared to that of the southwest composite, leading to a relatively weak mean VWS $\left(15.1 \mathrm{~m} \mathrm{~s}^{-1}\right)$, but still retaining a large value of mean $\operatorname{EFC}\left(24.8 \mathrm{~m} \mathrm{~s}^{-1} \mathrm{~d}^{-1}\right)$. The whole pattern resembles an "S". This "S"-shaped flow has been reported to favor the intensification process of TCs

Table 2. Characteristics of the eight composites for all positive interaction samples. Composites were selected according to the averaged 200-hPa wind direction within $500 \mathrm{~km}$ of TCs. Note that the mean SSTs were computed using samples that were over the ocean. Mean SST and VWS are averaged within $500 \mathrm{~km}$ and the mean EFC and inertial stability $\overline{\zeta_{a}}$ are averaged over the $300-600 \mathrm{~km}$ radial bands.

\begin{tabular}{lcccccccr}
\hline Wind direction & $\begin{array}{c}\text { No. } \\
\text { of samples }\end{array}$ & $\begin{array}{c}\text { Intensifying } \\
(\%)\end{array}$ & $\begin{array}{c}\text { No change } \\
(\%)\end{array}$ & $\begin{array}{c}\text { Weakening } \\
(\%)\end{array}$ & $\begin{array}{c}\text { Mean SST } \\
\left({ }^{\circ} \mathrm{C}\right)\end{array}$ & $\begin{array}{c}\text { Mean VWS } \\
\left(\mathrm{m} \mathrm{s}^{-1}\right)\end{array}$ & $\begin{array}{c}\text { Mean EFC } \\
\left(\mathrm{m} \mathrm{s}^{-1} \mathrm{~d}^{-1}\right)\end{array}$ & $\begin{array}{c}\text { Mean } \overline{\zeta a} \\
\left(10^{-5} \mathrm{~s}^{-1}\right)\end{array}$ \\
\hline West & 926 & 29.5 & 41.4 & 29.2 & 20.9 & 21.4 & 26.0 & 8.7 \\
Northwest & 210 & 37.1 & 47.1 & 15.7 & 25.1 & 13.3 & 19.9 & 7.7 \\
North & 86 & 34.9 & 44.2 & 20.9 & 27.8 & 8.4 & 15.6 & 7.2 \\
Northeast & 69 & 40.2 & 36.2 & 21.7 & 28.7 & 10.0 & 14.6 & 6.8 \\
East & 17 & 11.8 & 76.5 & 11.8 & 28.4 & 11.3 & 13.2 & 6.4 \\
Southeast & 44 & 11.4 & 47.7 & 40.9 & 24.3 & 9.8 & 16.0 & 9.7 \\
South & 329 & 18.8 & 37.7 & 43.5 & 21.1 & 15.1 & 24.8 & 10.4 \\
Southwest & 1359 & 21.4 & 37.5 & 41.1 & 20.2 & 20.1 & 26.9 & 9.7 \\
\hline
\end{tabular}


(a)

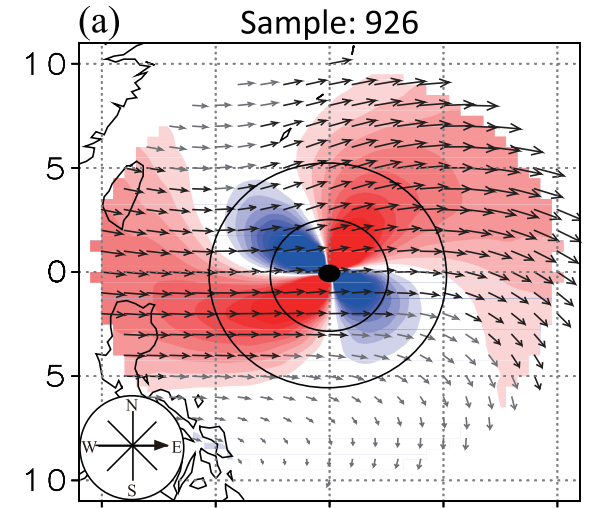

(c)
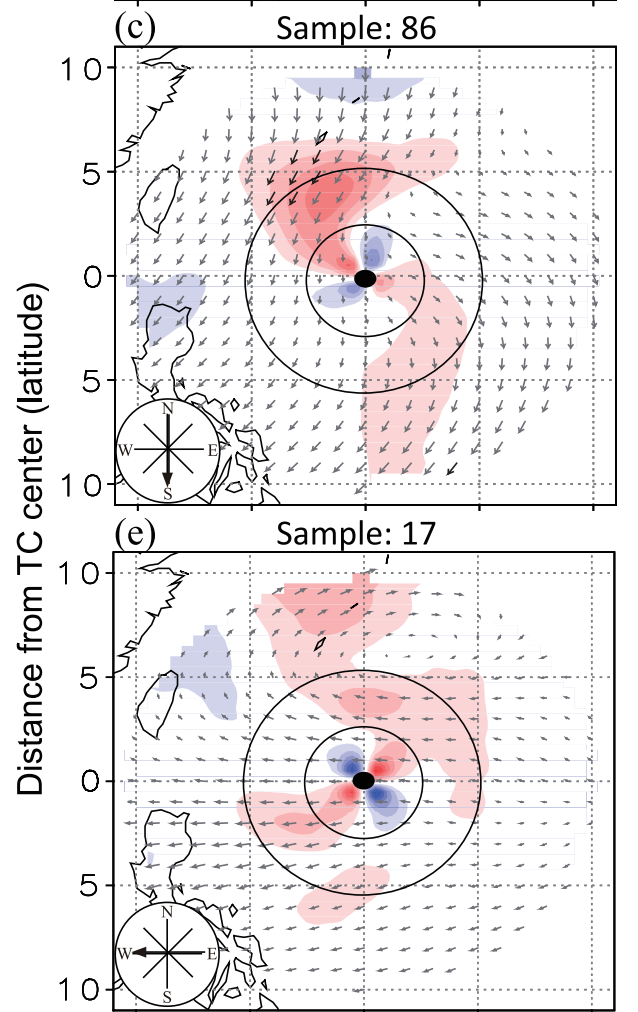

(g) Sample: 329

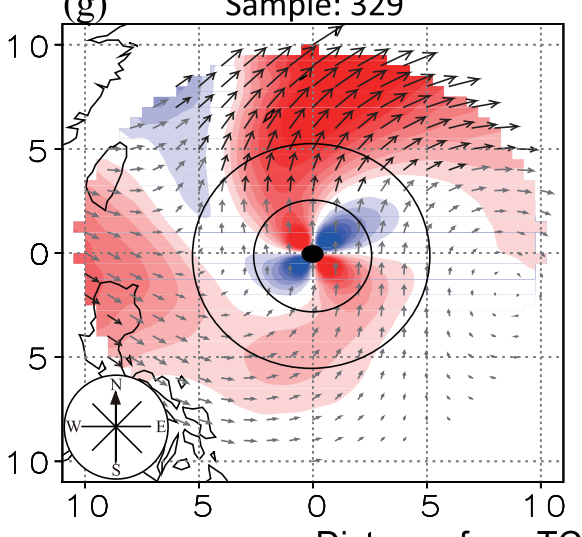

(b) Sample: 210

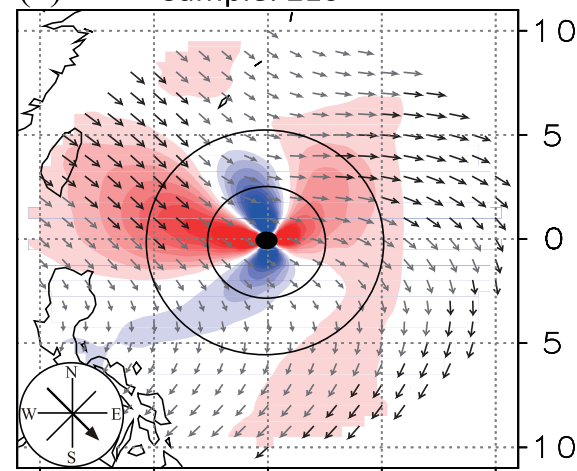

(d)
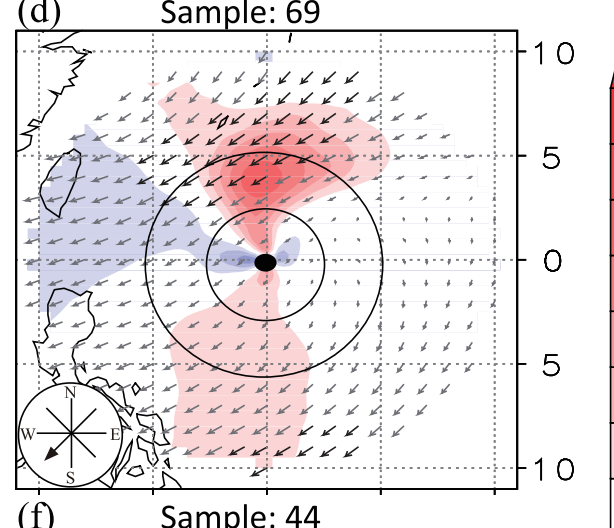

(f)

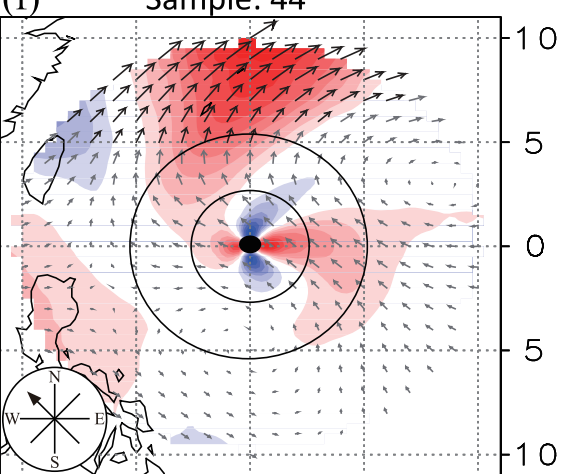

(h) Sample: $1359 \quad \overrightarrow{40}$

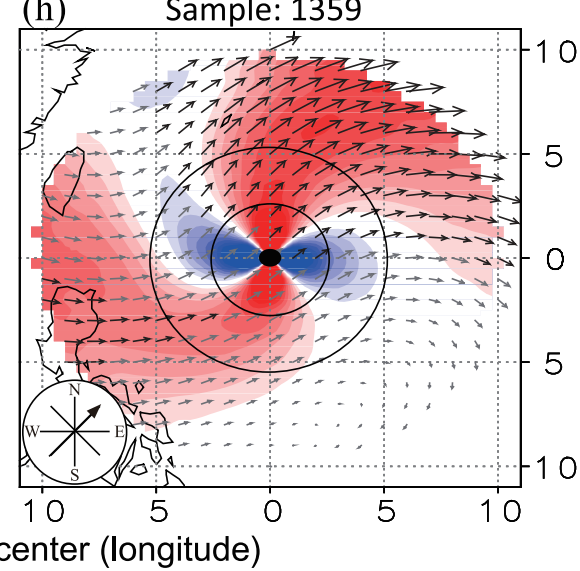

Fig. 5. Eight composites of 200-hPa storm-relative flow (arrows; $\mathrm{m} \mathrm{s}^{-1}$ ) and local EFC (shaded, $\mathrm{m} \mathrm{s}^{-1} \mathrm{~d}^{-1}$ ) for all positive interaction samples. Black bold arrows indicate wind speeds $>15 \mathrm{~m} \mathrm{~s}^{-1}$. Samples are grouped according to the averaged wind direction (shown in the lower-left corner of each panel) within $500 \mathrm{~km}$ of TCs. The composites were produced in cylindrical coordinates on a sphere, but the results were interpolated onto $1^{\circ} \times 1^{\circ}$ grids for a better visual. Black dots indicate TC centers and two black concentric circles in each panel show $300 \mathrm{~km}$ and $600 \mathrm{~km}$ radii from TC centers. The background map is shown for reference only. 
during the extratropical transition stage (Qian et al., 2011). However, from the statistical point of view, only $18.8 \%$ of the samples intensified and more than $43 \%$ of the samples decayed. The northwest composite (Fig. 5b), which is the least among those composites (southwest, west, south and northwest) related to westerlies, contains only 204 samples. The flow features are quite similar, except that the inflow jet shifts to the northwest and the outflow jet shifts to the southeast.

These four types, generally related to the upper-level westerly wind, contain more samples than the remaining four. A more interesting signature other than the trough in these composites is that there are two jets in the vicinity of the TC, like a dipole, resulting in an obvious azimuthal wavenumber2 structure of eddy flux convergence. Their roles can be clearly identified by the local EFC in which no azimuthal average was calculated in Fig. 5: the two jets bring in two local EFC maxima (red areas) with respect to the TC center, regardless of jet directions (i.e., inflow or outflow). However, inside the trough regions (northwest quadrants between two jets), only negative local EFC (blue area) is found, and thus the troughs act to reduce the EFC when averaging within the $300-600 \mathrm{~km}$ radial band. Therefore, this double-jet signature, with the outflow anticyclonic curved and the inflow cyclonic curved, results in a large EFC value rather than the traditional trough signature. These two jets cannot be ascribed to the interaction between the TC and trough because there would be such a flow pattern even without a TC. That is why their influence is also viewed as external (e.g., Molinari and Vollaro, 1989).

The remaining four composites of north, northeast, east and southeast (Figs. 5c-f) have relatively fewer samples. Such few samples may not produce a statistically significant percentage of intensifying or weakening samples, especially for the east composite. It seems that these interactions occur at lower latitudes where the prevailing upper-level wind has an easterly component (Figs. 5c-f) rather than synoptic waves embedded in westerlies. These TC samples are probably in their early stage as TDs because the underlying SST is relatively high (above $27^{\circ} \mathrm{C}$, except that of the southeast composite). The previously mentioned double-jet signature also applies to these composites, except that the jets are much weaker, but the traditional trough pattern can barely be identified in these four composites.

Figure 6 shows the local inertial stability, i.e., $\zeta_{\mathrm{a}}$ superposed by the composite wind fields. In terms of local inertial stability, the role of each jet, located in different quadrants, could be different because relatively weak inertial stability over a certain quadrant of the TC could minimize the energy expenditure and facilitate the formation of an outflow channel in that quadrant (Rappin et al., 2011). From Fig. 6 we can see that all composite $\zeta_{\mathrm{a}}$ values are positive, although this may not be true for each individual sample. Thus, the composite wind flows are generally inertially stable. However, the azimuthal distribution of $\zeta_{\mathrm{a}}$ is quite uneven, showing obvious azimuthal wavenumber- 1 asymmetry. In general, the jet cores separate the $\zeta_{\mathrm{a}}$ into two parts-a larger part that is more inertially stable and locates inside the troughs, and a smaller part that is less stable. As can be seen from Fig. 6, the roles of the two jets are different in terms of inertial stability because the outflow jet is roughly within the less inertially stable region while the inflow jet is inside the more stable region. Given roughly the same environmental EFC forcing, the contribution of the outflow jet to the TC's axisymmetric secondary circulation is more significant than that of the inflow jet due to weaker inertial stability. Besides, as mentioned previously, smaller axisymmetric $\overline{\zeta_{\mathrm{a}}}$, after azimuthal averaging, indicates less resistance to the environmental EFC forcing and a stronger vortex response in the TC's radialvertical circulation. These conditions apply to the four groups of north, northeast, east, and southeast composites (Figs. 6cf), as these interactions occur at relatively low latitudes (Table 2 ), characterized by smaller planetary vorticity.

It is worth mentioning that there are some similarities between the eight flow patterns identified here and those proposed in previous studies. For example, Holland and Merrill (1984) showed the manner in which a cyclone interacts with the subtropical westerlies and produces a poleward outflow channel (see their Fig. 15, and notice that their illustration is in the Southern Hemisphere). They also emphasized the effect of the subtropical jet on enhancing the TC's outflow, which is quite similar to Figs. 5f and g shown here. Figures 5f and g are similar to Holland and Merrill's (1984) illustration in the sense that the subtropical jet is located just north of the TC center and the TC keeps a certain distance away from the jet (heavy black arrows). However, the maximum radius is $\sim 1000 \mathrm{~km}$ in the composites. Therefore, the complete nature of the jet in Fig. 5f cannot be shown. Notice that there are also jet maxima just to the north of the TC center in Fig. 5h, but TCs are already inside the westerly jet core with large VWS, which is different from the picture of Holland and Merrill (1984). Another example was given by McTaggart-Cowan et al. (2006), who presented a conceptual model of tropical transition in a dipole-blocking environment (see the right-hand column of their Fig. 2). Their flow pattern is quite similar to Figs. 5f and g shown here, in which the anticyclone to the northeast of the TC center develops intensively so that it is gradually cut off from the westerlies and forms a cutoff blocking. Such a blocking flow pattern, as noted by McTaggart-Cowan et al. (2006), would facilitate a midlatitude vortex precursor transiting into a TC (known as tropical transition), whereas the similar flow pattern in Fig. $5 \mathrm{~g}$ is likely to facilitate the transition of a TC into an EC (known as extratropical transition), depending on the accompanying VWS and underlying SST. A third example is the typical tropical upper-tropospheric trough (TUTT) flow pattern given by Fitzpatrick et al. (1995). The TUTT pattern (see their Fig. 2), in which the axes of the shallow troughs lie in the northeast quadrants and also tilt northeast, resembles those of the north and northeast composites shown in Figs. $5 \mathrm{c}$ and $\mathrm{d}$ here.

The EFC criterion, as shown here, actually does not guarantee the occurrence of westerly troughs but reflects azimuthal shears of the flow crossing the TC's center, especially those induced by wavenumber- 1 asymmetry. Curvatures of 
(a)

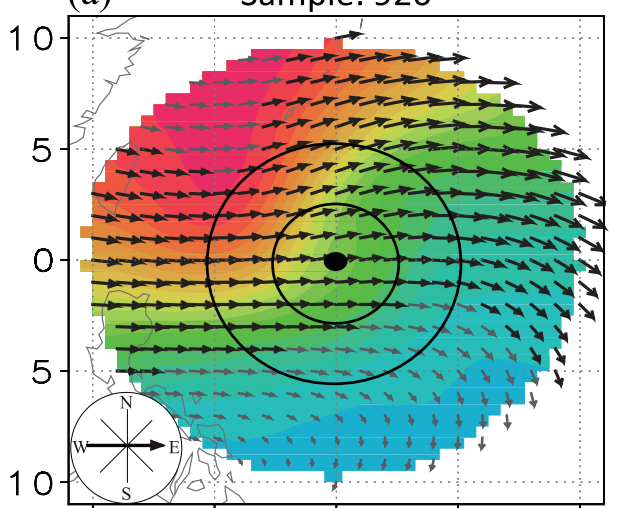

(c)
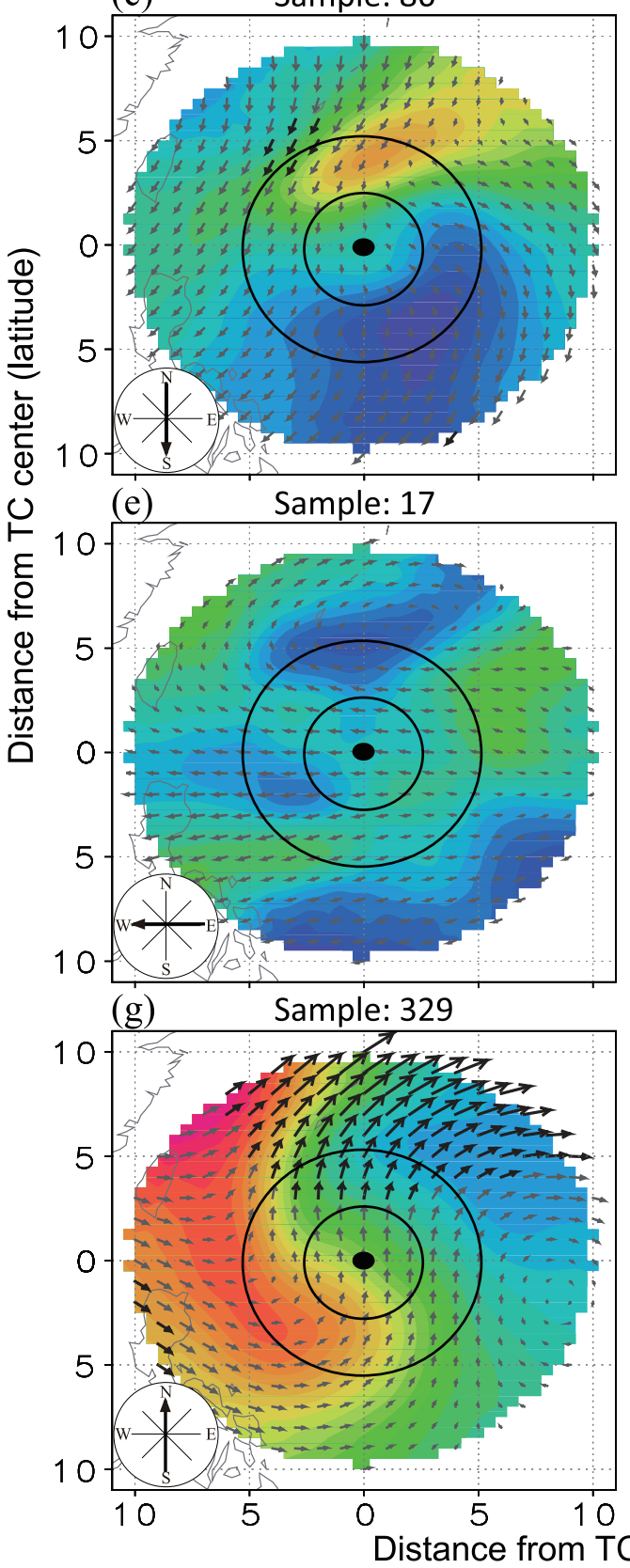

(b) Sample: 210

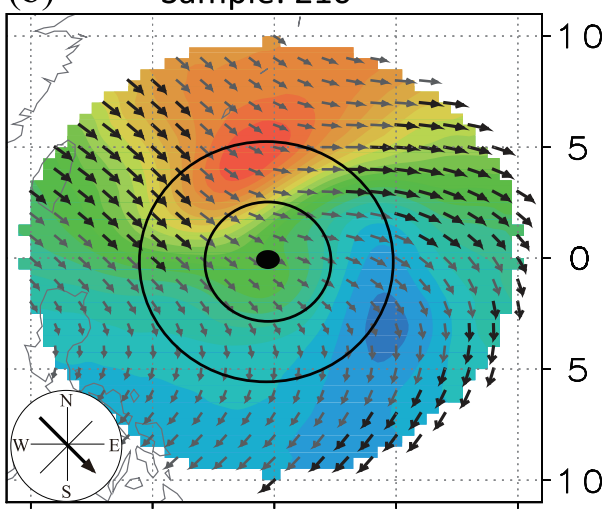

(d) Sample: 69

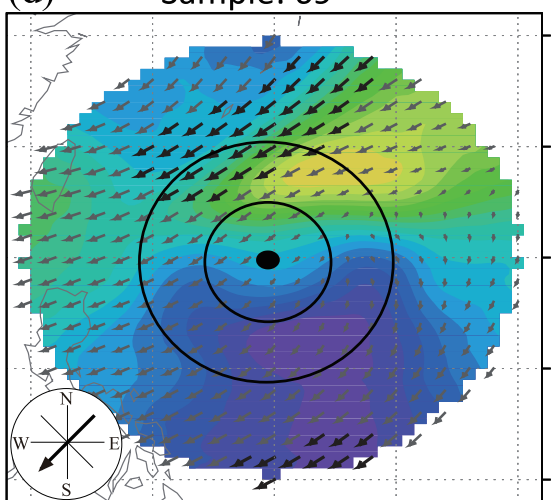

(f)

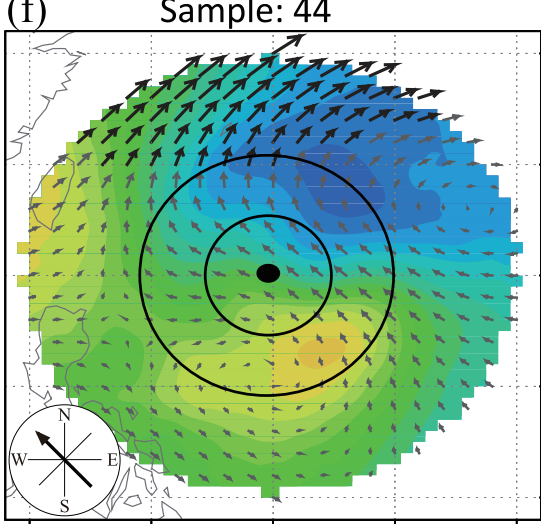

(h)

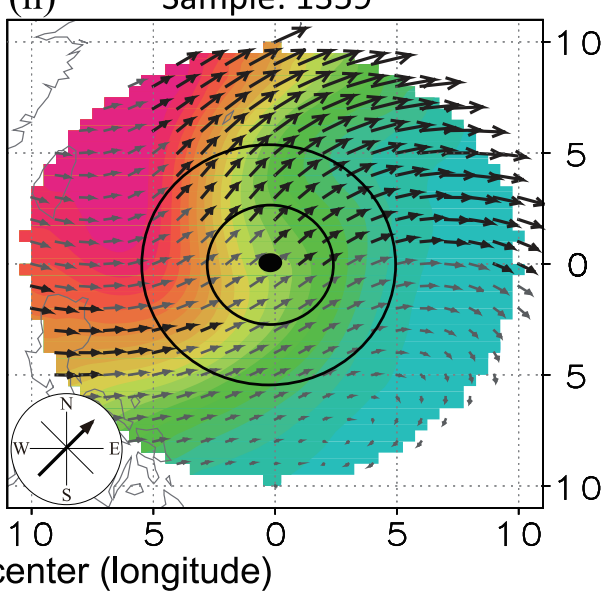

Fig. 6. As in Fig. 5 but for local absolute vorticity $\zeta_{\mathrm{a}}$ (shaded; $10^{-5} \mathrm{~s}^{-2}$ ), defined in Eq. (2), as a proxy for local inertial stability. 
the flow (i.e., cyclonic curved inflow and anticyclonic curved outflow) may eventually decide the sign of EFC. It was also found that extremely large EFC only occurs in those environments with large wind magnitude (e.g., Fig. 4), usually inside westerly jets. Therefore, we emphasize the feature of a double-jet signature of upper-level flows when interaction occurs because it is the curved jets, rather than troughs, that import large EFC from the environment to the TC (Fig. 5), resulting in significant upper-level asymmetric forcing on the TC's axisymmetric secondary circulation.

\section{Environmental factors and TC intensity change}

\subsection{Relationship between EFC and other environmental factors}

Studies (e.g., Wu and Cheng, 1999; Chen et al., 2015) have shown that SST, VWS and EFC are three large-scale environmental factors affecting TC intensity. Their effects may overlap to result in a more favorable environment for TC strengthening, or be cancelled to lead to a less favorable one. Therefore, the relationship between EFC and other environmental factors was investigated, based on the 25-year dataset.

According to the SEB theory (section 2.2), large EFC would favor TC intensification. However, from Table 2 we can see that there is no simple "large EFC-large percentage of intensifying samples (or small percentage of decaying samples)" relationship, primarily due to two reasons. One reason is that larger EFC is generally accompanied by greater inertial stability (see the last two columns in Table 2). Inertial stability is a measure of the resistance of vortex responses to eddy forcing. High inertial stability means that strong forcing will result in limited responses. As a TC intensifies, especially when doing so rapidly, it requires low inertial stability (e.g., Rappin et al., 2011); the higher inertial stability shown here is partially responsible for reducing the positive effect of large EFC. Another reason is that TC intensity change is also controlled by two other environmental factors, i.e., SST and VWS. Larger EFC corresponds to lower SST and larger VWS so that EFC's positive effect on TC intensification is generally cancelled out by the negative effect of low SST and large VWS. All these facts can be explained by the variation in the latitude at which the TC is located, as a higher latitude usually means larger $\bar{\zeta}_{\mathrm{a}}$ (larger planetary vorticity $f$ ), lower SST, larger wind speed, and thus larger VWS and EFC.

Figure 7 shows scatter plots of EFC versus SST for TC samples grouped by different TC stages. For the TD, TS and TY types (Figs. 7a-c), a large proportion of samples occur in a favorable environment of a warm ocean $\left(\mathrm{SST}>25^{\circ} \mathrm{C}\right.$ ). There are only a few samples whose underlying SSTs are lower than $20^{\circ} \mathrm{C}$. The mean EFCs are slightly larger than 0 , showing a skewness towards a positive EFC environment. For the EC type samples, as they have actually moved over cold water at higher latitudes, SST is much lower than those of TDs, with a mean of $\sim 16^{\circ} \mathrm{C}$ and large variance. Their mean EFC is close to $20 \mathrm{~m} \mathrm{~s}^{-1} \mathrm{~d}^{-1}$, already exceeding the threshold of positive interaction, which means that ECs are essentially different from other TC types and large upperlevel EFC may be one of their characteristics. For all four types, it was found that the magnitude of EFC does not exceed $40 \mathrm{~m} \mathrm{~s}^{-1} \mathrm{~d}^{-1}$ if SST is higher than $25^{\circ} \mathrm{C}$. However, extremely large EFCs occur frequently in lower SST environments. For the TS and TY samples, the highest EFC, at $80-120 \mathrm{~m} \mathrm{~s}^{-1} \mathrm{~d}^{-1}$, was in the SST range of $15^{\circ} \mathrm{C}-20^{\circ} \mathrm{C}$. For the EC samples, this was even more prominent, in that EFC could reach above $120 \mathrm{~m} \mathrm{~s}^{-1} \mathrm{~d}^{-1}$ when SST was below $15^{\circ} \mathrm{C}$.

The relationship between VWS and EFC (Fig. 8) is the reverse of that between SST and EFC. Extremely large EFC usually occurs in a large VWS environment, but large VWS does not necessarily induce large EFC, as EFC also requires some curvature of the wind flow. According to the SEB theory, large EFC is usually expected to significantly enhance a TC's axisymmetric outflow, strengthen the updraft near the TC center, and lead to TC intensification. Here, it is shown that extremely large EFC $\left(>40 \mathrm{~m} \mathrm{~s}^{-1} \mathrm{~d}^{-1}\right)$ is usually accompanied by relatively low SST and strong VWS, so that the positive contribution of EFC to a TC's intensification is cancelled out by the negative contribution of the low SST and strong VWS. Therefore, EFC larger than $40 \mathrm{~m} \mathrm{~s}^{-1} \mathrm{~d}^{-1}$ does not guarantee TC intensification. On the contrary, such an environment would destroy a TC's warm-core structure and possibly lead to its transformation into an EC.

\subsection{Environmental factors and TC intensity change}

In order to investigate the relationship between environmental factors and TC intensity change, intensifying and weakening TC samples were selected. Generally, whether a TC is intensifying or weakening can be identified from the sign of pressure changes. Taking into account that the pressure records in the RSMC best track dataset are discrete, with a minimum interval of $2 \mathrm{hPa}$, and that the records may contain some uncertainties, records with $|\Delta p|>3 \mathrm{hPa}$ were considered as the threshold for intensity change.

Scatter plots of the intensifying $(\Delta p<-3 \mathrm{hPa})$ and weakening $(\Delta p>3 \mathrm{hPa}) \mathrm{TC}$ samples and their environmental factors are shown in Fig. 9. For the intensifying samples (righthand column in Fig. 9), there are obvious scatter clouds within regions of SST $>25^{\circ} \mathrm{C}$ (the circle in Fig. 9b) and VWS $<15 \mathrm{~m} \mathrm{~s}^{-1}$ (the circle in Fig. 9d). These clouds consist of samples from classic TC types, i.e., TD, TS and TY, while the EC samples are generally located outside the clouds (see Figs. $7 \mathrm{~d}$ and $8 \mathrm{~d}$ ). For the classic TC types, environments with $\mathrm{SST}>25^{\circ} \mathrm{C}$ and VWS $<15 \mathrm{~m} \mathrm{~s}^{-1}$ are generally known as necessary conditions for their maintenance or development. Their EFC magnitudes seldom exceed $25 \mathrm{~m} \mathrm{~s}^{-1} \mathrm{~d}^{-1}$. For the EC-type samples outside the dense clouds, it is surprising that all intensifying samples are in a positive EFC environment. Other factors, such as VWS or SST, place no obvious constraint on the distribution of these samples. This can be explained by the fact that ECs are baroclinic systems and gain energy primarily from baroclinic instability, while classic TC types (TD, TS and TY) gain energy mainly from latent (dia- 
(a)

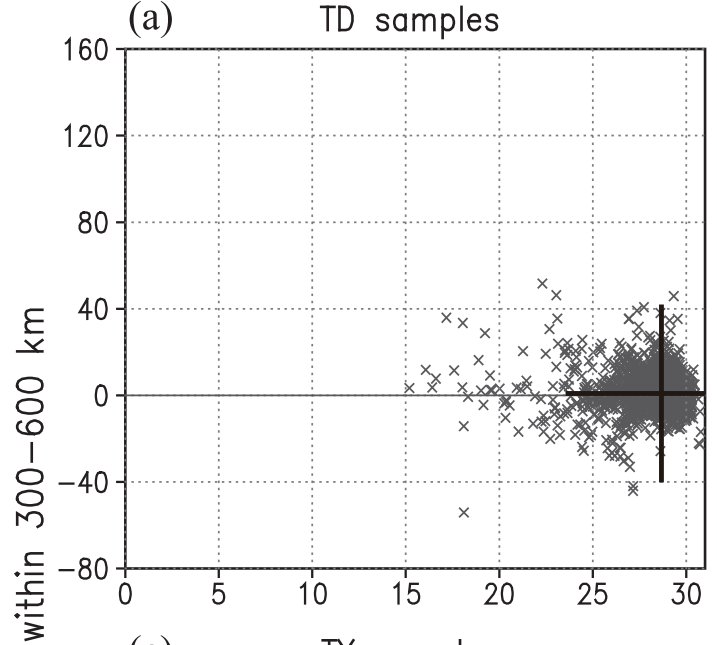

(c)

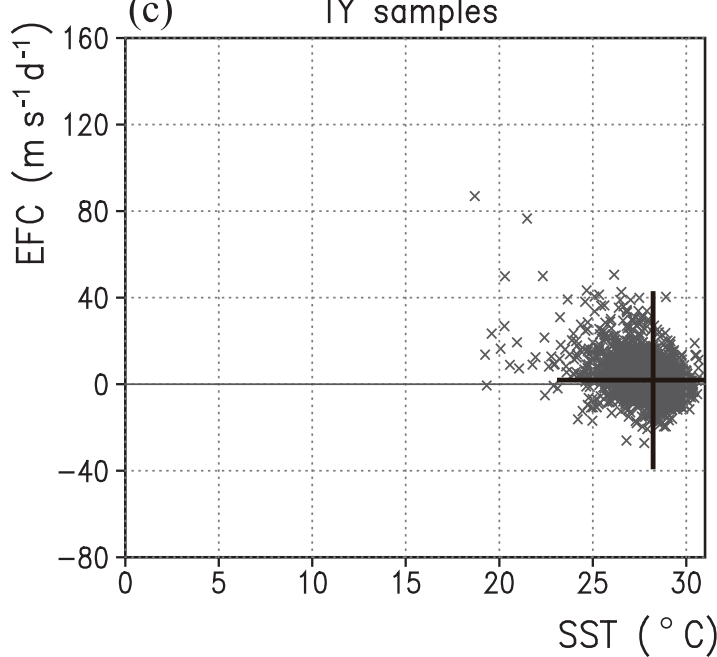

(b)

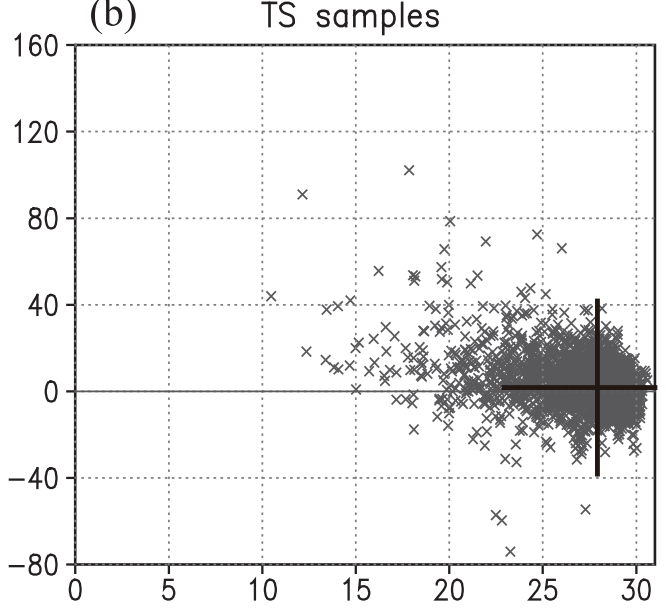

(d)

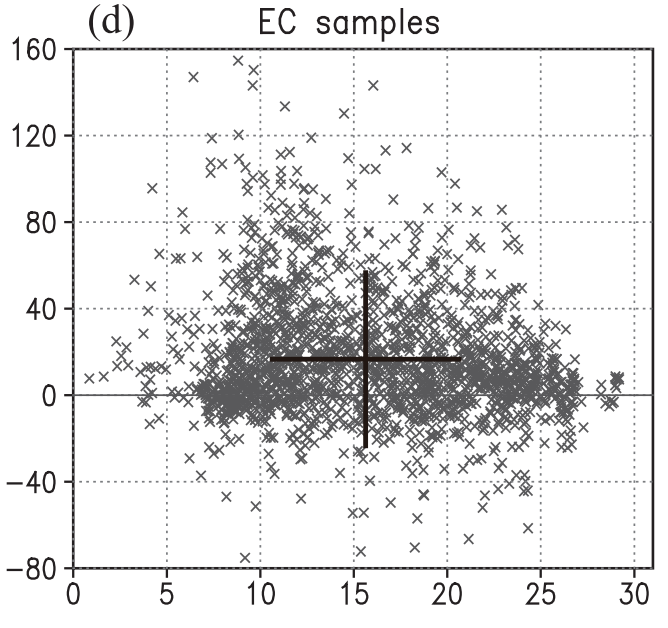

Fig. 7. Scatter diagrams of SST $\left({ }^{\circ} \mathrm{C}\right)$ averaged within $500 \mathrm{~km}$ versus $200-\mathrm{hPa}$ EFC $\left(\mathrm{m} \mathrm{s}^{-1} \mathrm{~d}^{-1}\right)$ averaged within 300-600 km for (a) TD, (b) TS, (c) TY and (d) EC samples. Samples of landing or near land (within $200 \mathrm{~km}$ ) are excluded since no reliable SST can be obtained, yielding 15999 samples. Black crosses indicate the mean EFC and SST for each panel.

batic) heating. For weakening samples (left-hand column in Fig. 9), the scatter clouds are not as concentrated as their intensifying counterparts, and there are no obvious features in selecting an environment.

Since ECs are different from other TC types in response to their environments, we should exclude them for further study. It is also known that large EFC may not correlate with simultaneous TC intensity, but has a lead-lag impact on later intensity change (e.g., Molinari and Vollaro, 1990). Therefore, it is more appropriate to define an interaction event as three consecutive six-hourly samples experiencing at least 10 $\mathrm{m} \mathrm{s}^{-1} \mathrm{~d}^{-1}$ EFC. This definition groups those interaction samples into events and excludes samples that only once or twice exceed the $10 \mathrm{~m} \mathrm{~s}^{-1} \mathrm{~d}^{-1}$ threshold, which has little persistent effect on TC intensity. Samples with maximum sustained wind speed of $<17.2 \mathrm{~m} \mathrm{~s}^{-1}$ were not considered, so as to exclude most EC samples, since ECs are different from other types. This procedure also excluded TDs, as there are large uncertainties in intensity records of weak intensity samples (e.g., Barcikowska et al., 2012).

There were only 166 interaction events identified using the above constraints. The corresponding statistics of these events are listed in Table 3. The number of weakening events was twice as many as intensifying events. Therefore, drawing the simple conclusion that EFC $>10 \mathrm{~m} \mathrm{~s}^{-1} \mathrm{~d}^{-1}$ may facilitate TC intensification is still not possible. Other factors, such as SST and VWS, seem more favorable for intensification. After excluding EC samples, the mean EFCs for both intensifying and weakening events remains at $\sim 25 \mathrm{~m} \mathrm{~s}^{-1} \mathrm{~d}^{-1}$. This value can be viewed as a threshold, and if environmental EFC exceeds this value the positive effect of EFC would be offset by accompanying large VWS, as well as low SST, possibly leading to extratropical transition of TCs.

It is known that TCs usually weaken when close to land or are already landing. Thus, we added another condition that interaction events near land, or that had already landed, should be excluded. It is also known that, given an underlying SST, TCs have their maximum potential intensity (MPI). If a 
(a)

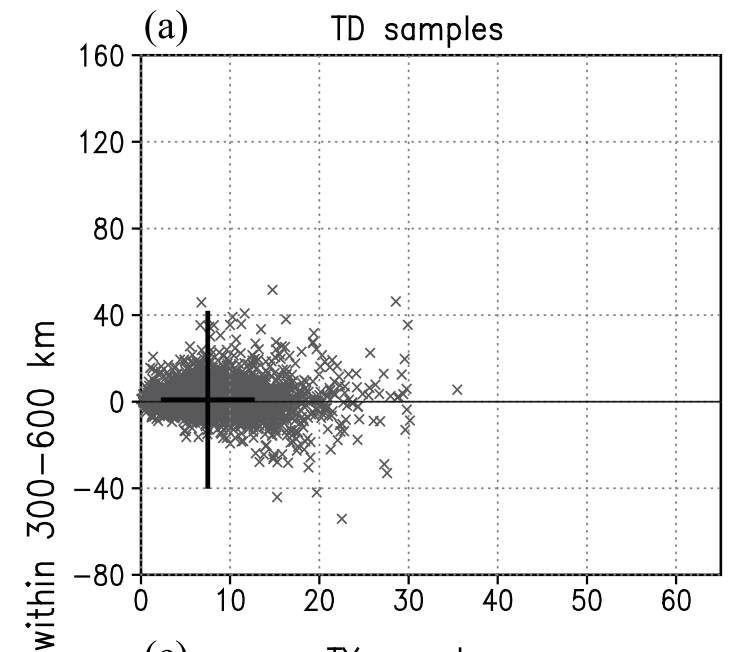

(c) TY samples

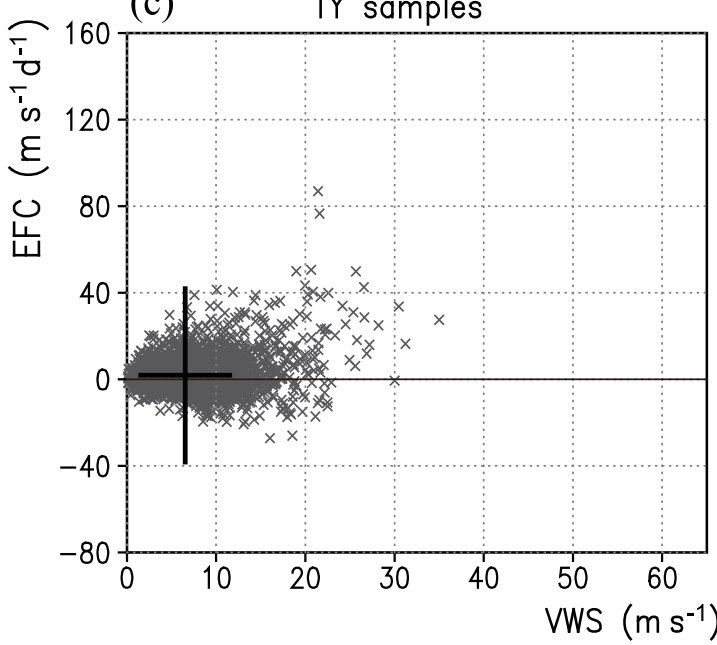

(b)

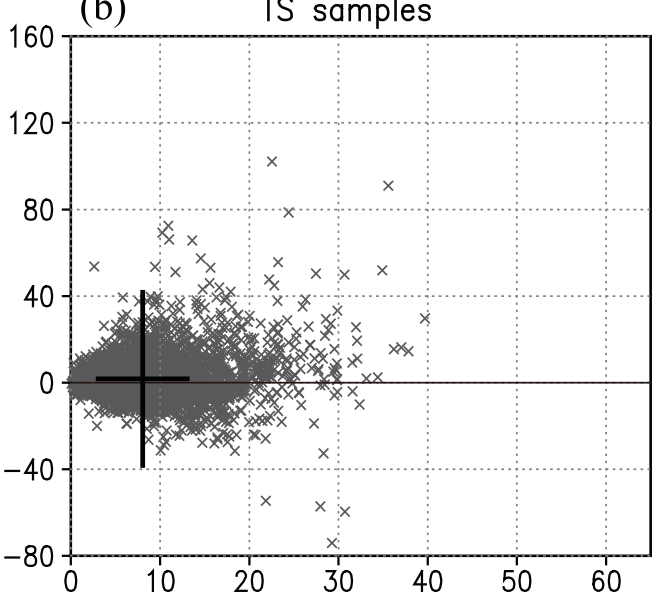

(d)

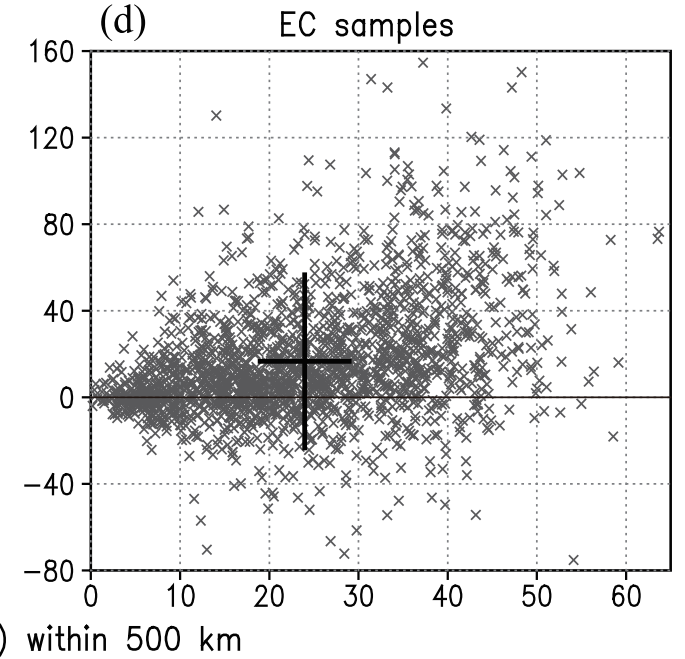

Fig. 8. As in Fig. 7 but for VWS $\left(\mathrm{m} \mathrm{s}^{-1}\right)$.

Table 3. Statistics of interaction events. Mean $\Delta p$ was estimated by the linear trend of SLP during the event using the least-squares fit method.

\begin{tabular}{lccccrrr}
\hline & & $\begin{array}{c}\text { No. } \\
\text { of events }\end{array}$ & $\begin{array}{c}\text { Mean SST } \\
\left({ }^{\circ} \mathrm{C}\right)\end{array}$ & $\begin{array}{c}\text { Mean VWS } \\
\left(\mathrm{m} \mathrm{s}^{-1}\right)\end{array}$ & $\begin{array}{c}\text { Mean EFC } \\
\left(\mathrm{m} \mathrm{s}^{-1} \mathrm{~d}^{-1}\right)\end{array}$ & $\begin{array}{c}\text { Mean SLP } \\
(\mathrm{hPa})\end{array}$ & $\begin{array}{c}\text { Mean } \Delta p \\
(\mathrm{hPa})\end{array}$ \\
\hline All interaction events & Intensifying & 55 & 26.6 & 9.4 & 26.5 & 981.1 & -1.4 \\
& Weakening & 111 & 24.5 & 15.2 & 23.5 & 973.2 & 5.0 \\
No-landing events & Intensifying & 50 & 26.8 & 9.6 & 18.3 & 980.6 & -1.4 \\
& Weakening & 67 & 25.1 & 13.7 & 20.1 & 970.4 & 4.5 \\
No-landing Have-potential events & Intensifying & 41 & 27.7 & 8.8 & 16.2 & 980.9 & -1.7 \\
& Weakening & 26 & 27.0 & 11.2 & 18.0 & 969.7 & 4.3 \\
\hline
\end{tabular}

TC is close to its MPI (usually measured as surface maximum wind speed), then any atmospheric environment is hostile to a TC's intensity (Merrill, 1988b). That is, there is no room for the TC to develop. We imposed the final constraint that TCs should be at least $30 \mathrm{~m} \mathrm{~s}^{-1}$ away from their MPI, which was estimated using the empirical relation given by Zeng et al. (2007) for the western North Pacific as:

$$
\mathrm{MPI}=A+B \exp \left[C\left(T_{\mathrm{s}}-T_{0}\right)\right],
$$

where $A=15.69 \mathrm{~m} \mathrm{~s}^{-1}, B=98.03 \mathrm{~m} \mathrm{~s}^{-1}, C=0.1806^{\circ} \mathrm{C}^{-1}$, $T_{\mathrm{S}}$ is SST, and $T_{0}=30.0^{\circ} \mathrm{C}$.
Table 3 shows that when the "no-landing" condition is added, the number of weakening events is greatly reduced from 111 to 67 , and the rate of SLP change is also reduced from $5 \mathrm{hPa}$ to $4.5 \mathrm{hPa}$ per six hours. After the "havepotential" condition is applied, the number of weakening events drops to 26, roughly half of that of intensifying events. Besides, the intensifying rate increases from $1.4 \mathrm{hPa}$ to 1.7 $\mathrm{hPa}$ per six hours. However, even with these constraints, it is barely possible to draw the conclusion that higher EFC values will result in more intensifying events, because the mean EFC for an intensifying event is $16.2 \mathrm{~m} \mathrm{~s}^{-1} \mathrm{~d}^{-1}$, which is 
(a) Weakening samples $(\Delta p>3 \mathrm{hPa})$

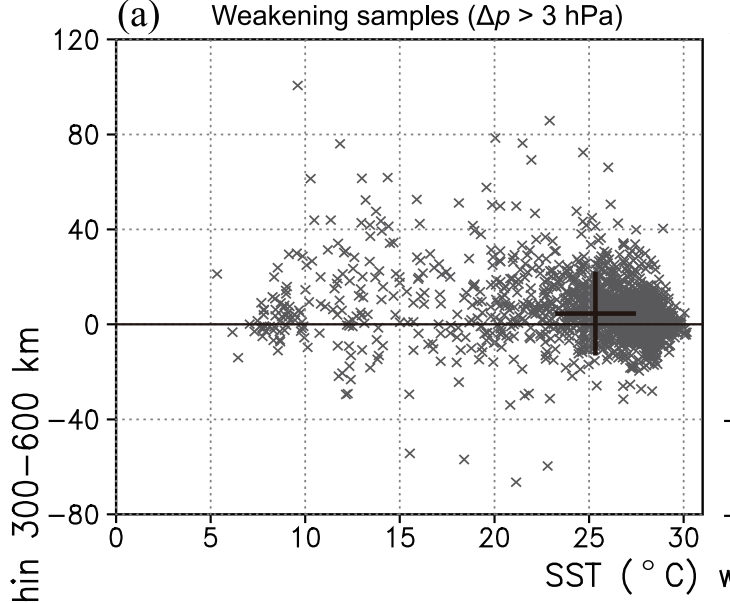

$\frac{5}{\frac{5}{3}}$

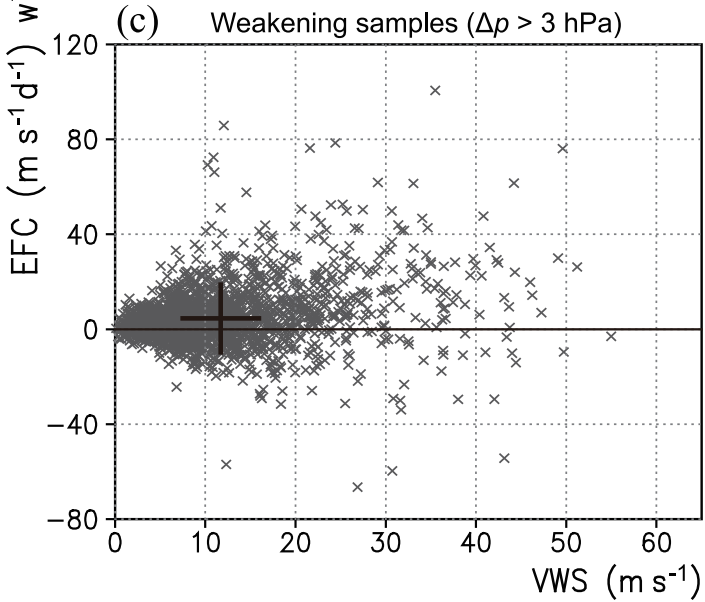

(b) Intensifying samples $(\Delta p<-3 \mathrm{hPa})$

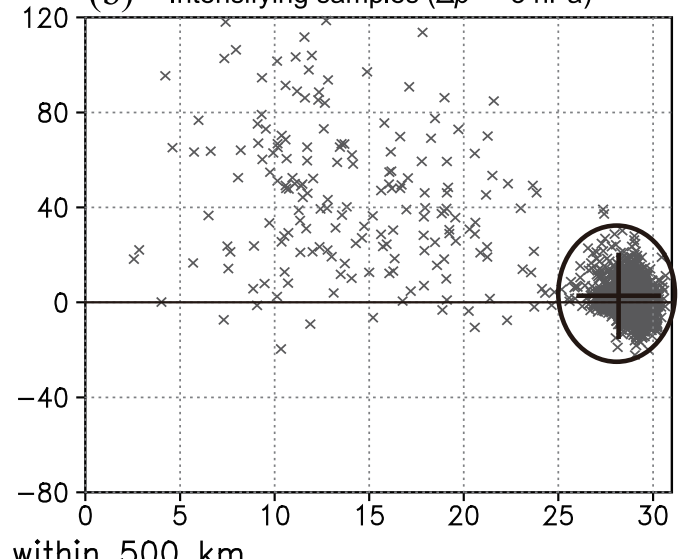

(d) Intensifying samples $(\Delta p<-3 \mathrm{hPa})$

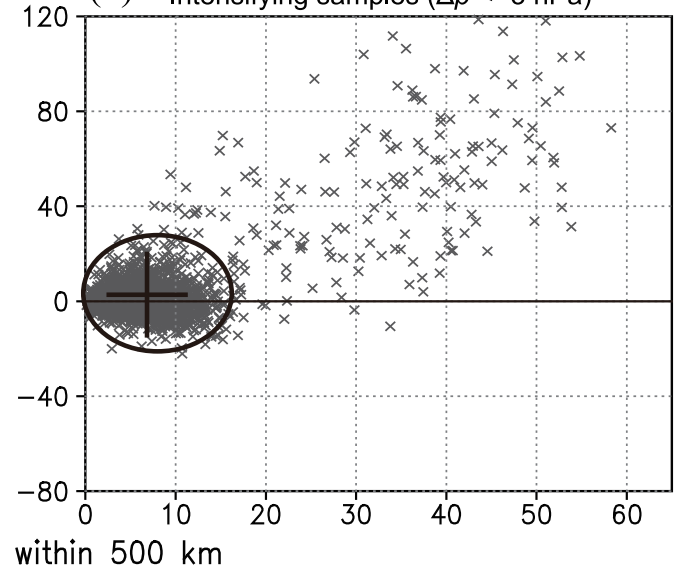

Fig. 9. Scatter diagrams of $(\mathrm{a}, \mathrm{b}) \mathrm{SST}\left({ }^{\circ} \mathrm{C}\right)$ and $(\mathrm{c}, \mathrm{d}) \mathrm{VWS}\left(\mathrm{m} \mathrm{s}^{-1}\right)$ averaged within $500 \mathrm{~km}$ versus 200-hPa EFC $\left(\mathrm{m} \mathrm{s}^{-1} \mathrm{~d}^{-1}\right)$ averaged within 300-600 km for (a, c) weakening samples $(\Delta p>3 \mathrm{hPa})$ and $(\mathrm{b}, \mathrm{d})$ intensifying samples $(\Delta p<-3 \mathrm{hPa}$ ). TC samples landing or near land (within $200 \mathrm{~km}$ ) are excluded since no reliable SST can be estimated. Black crosses indicate the mean VWS, SST and EFC for each panel. Black circles highlight the scatter clouds in panels (b, d).

relatively smaller than that of weakening events. Thus, regardless of these constraints, the intensifying events seem to be affected more by the SST and VWS rather than by EFC environments. As noted earlier, EFC exceeding the $25 \mathrm{~m} \mathrm{~s}^{-1}$ $\mathrm{d}^{-1}$ threshold is likely to induce very large VWS and low SST, and thus negative effects of the environment are more pronounced than positive ones, leading to weakening TCs or even deforming them into ECs. Here, EFC larger than this value tends to slow down the intensification rate or increase the possibility of filling a TC. So, we regard a moderate EFC environment, larger than $10 \mathrm{~m} \mathrm{~s}^{-1} \mathrm{~d}^{-1}$ but generally below $25 \mathrm{~m} \mathrm{~s}^{-1} \mathrm{~d}^{-1}$, as a relatively good environment for TC intensification.

\section{Conclusions}

A preliminary statistical survey of the interactions between TCs and upper-level environmental flows over the western North Pacific is presented in this paper using a 25year (1987-2011) best track dataset and ERA-Interim data.
Interactions are defined as the magnitude of EFC exceeding $10 \mathrm{~m} \mathrm{~s}^{-1} \mathrm{~d}^{-1}$. According to this definition, it was found that $17.7 \%$ of 21685 six-hourly TC samples experienced interaction: $14 \%$ positive and $3.7 \%$ negative, according to their EFC signs. These interactions were frequently identified around Japan, where TCs recurve into midlatitude westerlies.

Six extreme interaction cases showed that negative (positive) interactions are characterized by upper-level northwest (southwest) cross-center wind associated with the upstream (downstream) flow of a trough. Extreme negative EFC values reach $-70 \mathrm{~m} \mathrm{~s}^{-1} \mathrm{~d}^{-1}$, whose magnitude is only half that of positive ones $\left(120 \mathrm{~m} \mathrm{~s}^{-1} \mathrm{~d}^{-1}\right)$. These negative interactions, arising from slightly modifying the interaction definition, as compared to previous studies, also provides new features when EFC is negative. This kind of interaction also relates to upper-level troughs (Figs. $4 \mathrm{a}-\mathrm{c}$ ). The main difference is that the TC locates upstream of the trough, rather than downstream as in traditionally defined (i.e., positive) interaction.

Composite analysis showed that positive interactions are characterized by an apparently curved upper-level crossing- 
center flow that consists of two jets. These two jets, identified in the present study through analyzing localized EFC, are a more important signature than the traditionally proposed trough pattern because large positive local EFC comes from these two jets rather than from the trough itself. Therefore, the interaction strength (e.g., EFC value) largely depends on the jet strength as well as their curvature. It is also shown that the outflow jet, exporting anticyclonic eddy angular momentum, is more important than the inflow one, importing cyclonic momentum, as the axisymmetric outflow enhances the outflow jet strength and lowers the adjacent inertial stability. That is why it is common to observe an enhanced outflow channel in the northern part of a TC without seeing any clear inflow channel (e.g., Rappin et al., 2011, Fig. 17).

Generally, according to the SEB theory, large positive $\mathrm{EFC}$ in the upper troposphere means that large cyclonic angular momentum is transported towards the TC from its vicinity and is thus favorable for TC axisymmetric outflow enhancement or even intensification. Previous studies (e.g., DeMaria et al., 1993) have already pointed out that such a positive effect will be offset by accompanying strong VWS and low SST. Here, we suggested separating the interactions into extreme cases (EFC larger than $25 \mathrm{~m} \mathrm{~s}^{-1} \mathrm{~d}^{-1}$ ) and moderate cases (10-25 $\mathrm{m} \mathrm{s}^{-1} \mathrm{~d}^{-1}$ ), according to Figs. 7 and 8. It was found that extreme interactions generally occur at high latitudes, meaning the environmental SST is very low and the VWS is quite strong. Besides, the inertial stability is also large at high latitudes (Table 2), which means that the positive effect of extremely large EFC will be scaled down. During such cases, the TC tends to transform into an EC. Only those ECs after transformation, which are less dependent on underlying high SST and low VWS, can survive or intensify in extremely large EFC environments (Fig. 9b). For moderate interactions that occur in the TS and TY stages, it is shown that their responses to EFC environments are quite different from that of the EC type. These traditional TCs can only survive in environments with small-to-moderate VWS, to maintain their warm core structures. The intensities of these TCs are also more sensitive to the underlying SST than EFC. Therefore, the "good trough/bad trough" issue still remains unclear for these traditional TCs.

The EFC threshold of $25 \mathrm{~m} \mathrm{~s}^{-1} \mathrm{~d}^{-1}$, empirically identified here, also distinguishes the interaction case of Hurricane Elena (1985) reported by Molinari and Vollaro (1989, 1990) as a canonical one, because this case only just reached the proposed upper limit of favorable interaction and then experienced a rapid intensification. It is also interesting to find cases over the western North Pacific that are similar to this canonical one, which may help us identify these "good" troughs. We selected TC cases with EFC larger than $20 \mathrm{~m}$ $\mathrm{s}^{-1} \mathrm{~d}^{-1}$ and VWS smaller than $10 \mathrm{~m} \mathrm{~s}^{-1}$ for at least one sixhour snapshot. There were only 153 samples ( $5 \%$ of positive interactions) that met these requirements, including several consecutive records in a single TC. Six representative interacting TCs - two during the formation stage (cases 1 and 2), two during the mature stage (cases 3 and 4), and two during the dissipation stage (cases 5 and 6)—were selected (shown in Fig. 10). As we can see, favorable interaction (cases $1-$ 4) does not indicate a westerly trough in the vicinity of the TC (e.g., cases 2 and 3). Even in the subtropics, flows with certain curvature can cause interaction. The enhanced EFC (green shaded areas) generally lead to subsequent intensifying processes. This is similar to the case of Hurricane Elena (1985), in which an EFC spike acted as a triggering effect for deepening the TC. Obviously, unfavorable interaction (cases 5 and 6) can be attributed to the increasing VWS and decreasing SST; although, at the interaction times (vertical gray lines), VWS does not exceed $10 \mathrm{~m} \mathrm{~s}^{-1}$. It also seems coincident that the 200-hPa flows in cases 5 and 6 resemble the non-intensifying pattern summarized by Merrill (1988b, Fig. 11 ), in which the anticyclones east of the TCs have closed streamlines.

Based on the conclusions drawn from the present study, as well as these representative cases, we can gain some insights into the "good" trough issue. Favorable interactions for ECs should be characterized by very large environmental EFC (at least above $25 \mathrm{~m} \mathrm{~s}^{-1} \mathrm{~d}^{-1}$ ), and the "larger is better" conclusion may apply for ECs regardless of the associated VWS and underlying SST. However, for traditional TC types, such as TDs, TSs or TYs, favorable interaction occurs with only moderate EFC above $10 \mathrm{~m} \mathrm{~s}^{-1} \mathrm{~d}^{-1}$, but generally below $25 \mathrm{~m} \mathrm{~s}^{-1} \mathrm{~d}^{-1}$, in favor of low VWS. This requires that TCs should keep a certain distance from the disturbed westerly jet (usually with a trough embedded upstream) and not penetrate into the jet core regions (such as in extreme interaction cases), as was schematically illustrated by Holland and Merrill (1984, Fig. 15) and also exemplified by Rappin et al. (2011, Fig. 17), so that their warm core structure will not be destroyed by persistently strong VWS. Besides, interaction should not last more than around two days, playing its role as a triggering effect for initiating wind-induced surface heat exchange (Emanuel, 1986) or the eyewall replacement cycle (Willoughby et al., 1982; Leroux et al., 2013), as long-lasting interaction will eventually bring persistent VWS, which is destructive to a TC's warm core. Finally, interaction should appear at low latitudes so that environmental inertial stability is relatively weak and thus the TC's axisymmetric secondary circulation is more sensitive to the upper-level asymmetric forcing.

The present study focused primarily on the dynamical effect of interaction in terms of EFC and inertial stability. In fact, a westerly trough approaching a TC will also import cold and dry air-also known as cold air intrusion. Such intrusion of cold and dry air will certainly alter the thermodynamic structure of a TC and exert some influence on its axisymmetric secondary circulation. Molinari and Vollaro (1990) found that the eddy heat flux induced by cold advection could contribute in the same direction as eddy momentum flux within $500 \mathrm{~km}$, but opposite outside. Therefore, Molinari et al. $(1995,1998)$ later analyzed interactions by adopting the Eliassen-Palm (EP) flux diagnostic, which has two components: eddy angular momentum flux (dynamical component) and eddy heat flux (thermodynamical component). As there is uncertainty as to whether the contribu- 

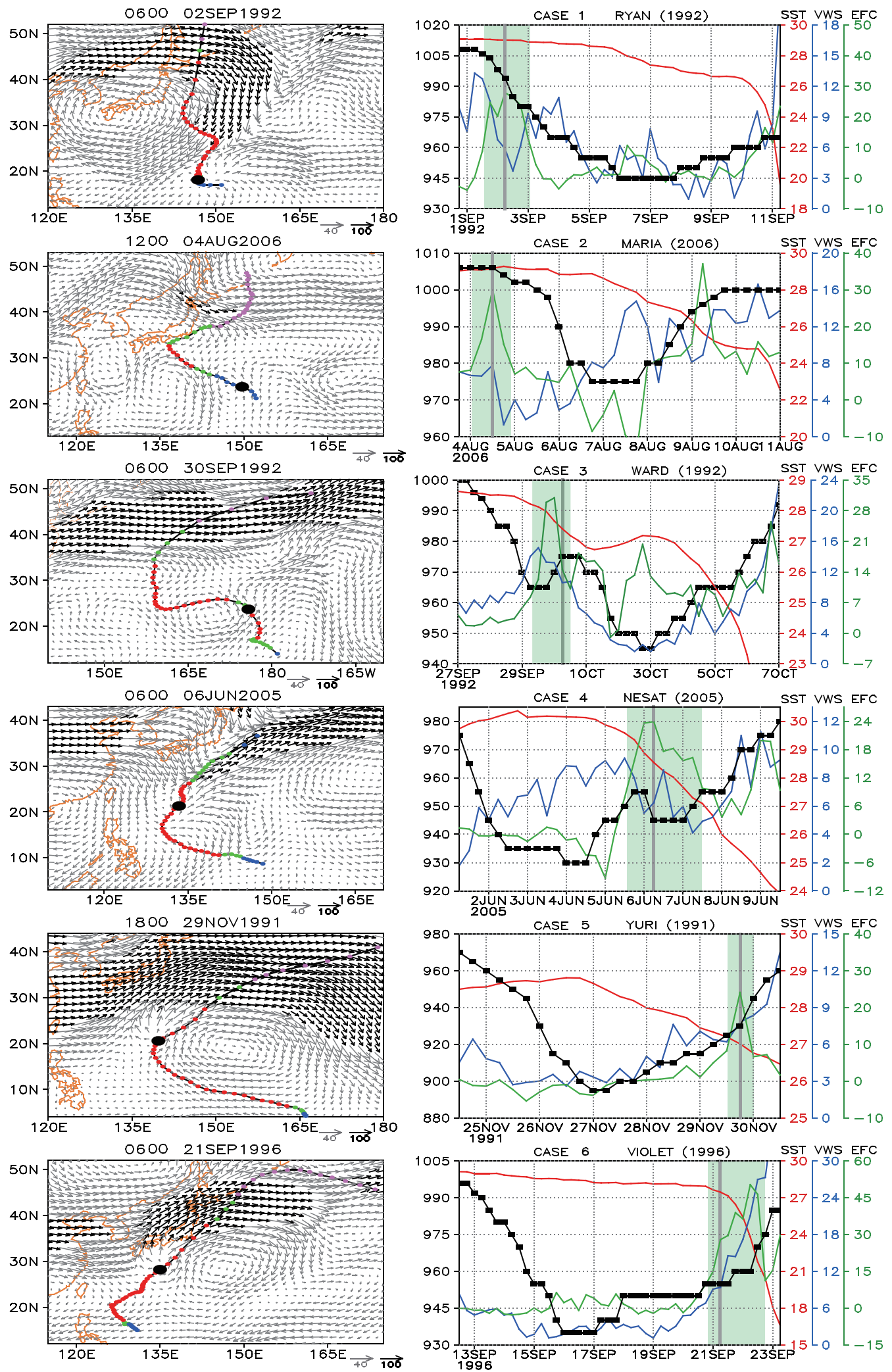

Fig. 10. Six selected interaction TCs (one row for each). The left-hand column shows the tracks for each case and 200-hPa flow fields (vectors; $\mathrm{m} \mathrm{s}^{-1}$ ) valid at interaction times labelled in the titles. Black dots show the TC positions at those times. The right-hand column shows the temporal evolution of TC intensity i.e., minimum SLP (black line; $\mathrm{hPa}$ ), as well as the three environmental factors of SST (red line; ${ }^{\circ} \mathrm{C}$ ), VWS (blue line; $\mathrm{m} \mathrm{s}^{-1}$ ) and EFC (green line; $\mathrm{m} \mathrm{s}^{-1} \mathrm{~d}^{-1}$ ). Vertical gray lines in the right-hand column indicate the time shown in the titles of the left-hand column. The green shaded areas emphasize the interaction periods of interest with EFC larger than the $10 \mathrm{~m} \mathrm{~s}^{-1} \mathrm{~d}^{-1}$ threshold. 
tions of these two components could be the same or opposite (e.g., Molinari and Vollaro, 1990; Qian et al., 2011), one way to address this issue is to use the EP flux diagnostic and its divergence. This diagnostic tool also requires the whole vertical structure of the TC, rather than a single upper-layer wind field, so that it will provide greater insights into TCenvironment interaction. Using such a diagnostic in a statistical study of interaction, rather than EFC alone, may help identify "good" troughs and favorable interactions.

Acknowledgements. This study was jointly sponsored by the National Natural Science Foundation of China (Grant Nos. 41205032, 41405048 and 41375050), China Special Fund for Meteorological Research in the Public Interest (Grant No. GYHY201406008), and the Guangdong Marine Disaster Emergency Response Technology Research Center (2012A032100004). The authors gratefully acknowledge the use of the High Performance Computing Cluster at the South China Sea Institute of Oceanology, Chinese Academy of Sciences.

\section{REFERENCES}

Barcikowska, M., F. Feser, and H. von Storch, 2012: Usability of best track data in climate statistics in the western North Pacific. Mon. Wea. Rev., 140, 2818-2830.

Bosart, L. F., and J. A. Bartlo, 1991: Tropical storm formation in a baroclinic environment. Mon. Wea. Rev., 119, 1979-2013.

Bosart, L. F., C. S. Velden, W. E. Bracken, J. Molinari, and P. G. Black, 2000: Environmental influences on the rapid intensification of Hurricane Opal (1995) over the Gulf of Mexico. Mon. Wea. Rev., 128, 322-352.

Bracken, W. E., and L. F. Bosart, 2000: The role of synopticscale flow during tropical cyclogenesis over the North Atlantic Ocean. Mon. Wea. Rev., 128, 353-376.

Challa, M., and R. L. Pfeffer, 1980: Effects of eddy fluxes of angular momentum on model hurricane development. J. Atmos. Sci., 37, 1603-1618.

Chan, J. C. L., F. M. F. Ko, and Y. M. Lei, 2002: Relationship between potential vorticity tendency and tropical cyclone motion. J. Atmos. Sci., 59, 1317-1336.

Chen, X. M., Y. Q. Wang, and K. Zhao, 2015: Synoptic flow patterns and large-scale characteristics associated with rapidly intensifying tropical cyclones in the South China Sea. Mon. Wea. Rev., 143, 64-87.

Choi, Y., K. -S. Yun, K. -J. Ha, K. -Y. Kim, S. -J. Yoon, and J. C. L. Chan, 2013: Effects of asymmetric SST distribution on straight-moving Typhoon Ewiniar (2006) and recurving Typhoon Maemi (2003). Mon. Wea. Rev., 141, 3950-3967.

Dee, D. P., and Coauthors, 2011: The ERA-Interim reanalysis: Configuration and performance of the data assimilation system. Quart. J. Roy. Meteor. Soc., 137, 553-597.

DeMaria, M., J. -J. Baik, and J. Kaplan, 1993: Upper-level eddy angular momentum fluxes and tropical cyclone intensity change. J. Atmos. Sci., 50, 1133-1147.

DeMaria, M., J. A. Knaff, and C. Sampson, 2007: Evaluation of long-term trends in tropical cyclone intensity forecasts. $\mathrm{Me}$ teor. Atmos. Phys., 97, 19-28.

Eliassen, A., 1952: Slow thermally or frictionally controlled meridional circulation in a circular vortex. Astrophysica Norvegica, 5, 19-60.
Elsberry, R. L., G. J. Holland, H. Gerrish, M. DeMaria, C. P. Guard, and K. A. Emanuel, 1992: Is there any hope for tropical cyclone intensity prediction?-A panel discussion. Bull. Amer. Meteor. Soc., 73, 264-275.

Emanuel, K. A., 1986: An air-sea interaction theory for tropical cyclones. Part I: Steady-state maintenance. J. Atmos. Sci., 43, 585-605.

Emanuel, K. A., 2000: A statistical analysis of tropical cyclone intensity. Mon. Wea. Rev., 128, 1139-1152.

Erickson, C. O., 1967: Some aspects of the development of Hurricane Dorothy. Mon. Wea. Rev., 95, 121-130.

Fitzpatrick, P. J., 1997: Understanding and forecasting tropical cyclone intensity change with the typhoon intensity prediction scheme (TIPS). Wea. Forecasting, 12, 826-846.

Fitzpatrick, P. J., J. A. Knaff, C. W. Landsea, and S. V. Finley, 1995: Documentation of a systematic bias in the aviation model's forecast of the Atlantic tropical upper-tropospheric trough: Implications for tropical cyclone forecasting. Wea. Forecasting, 10, 433-446.

Hanley, D. E., 2002: The evolution of a hurricane-trough interaction from a satellite perspective. Wea. Forecasting, 17, 916926.

Hanley, D., J. Molinari, and D. Deyser, 2001: A composite study of the interactions between tropical cyclones and uppertropospheric troughs. Mon. Wea. Rev., 129, 2570-2584.

Holland, G. J., and R. T. Merrill, 1984: On the dynamics of tropical cyclone structural changes. Quart. J. Roy. Meteor. Soc., 110, 723-745.

Hoskins, B. J., M. E. McIntyre, and A. W. Robertson, 1985: On the use and significance of isentropic potential vorticity maps. Quart. J. Roy. Meteor. Soc., 111, 877-946.

Kimball, S. K., and J. L. Evans, 2002: Idealized numerical simulations of hurricane-trough interaction. Mon. Wea. Rev., 130, 2210-2227.

Leroux, M. -D., M. Plu, D. Barbary, F. Roux, and P. Arbogast, 2013: Dynamical and physical processes leading to tropical cyclone intensification under upper-level trough forcing. $J$. Atmos. Sci., 70, 2547-2565.

Lewis, B. M., and D. P. Jorgensen, 1978: Study of the dissipation of Hurricane Gertrude (1974). Mon. Wea. Rev., 106, 1288 1306.

Li, Y., L. S. Chen, and X. T. Lei, 2006: Numerical study on impacts of upper-level westerly trough on the extratropical transition process of Typhoon Winnie (1997). Acta Meteorologica Sinica, 64, 552-563. (in Chinese)

Martin, J. D., and W. M. Gray, 1993: Tropical cyclone observation and forecasting with and without aircraft reconnaissance. Wea. Forecasting, 8, 519-532.

McTaggart-Cowan, R., L. F. Bosart, C. A. Davis, E. H. Atallah, J. R. Gyakum, and K. A. Emanuel, 2006: Analysis of Hurricane Catarina (2004). Mon. Wea. Rev., 134, 3029-3053.

Merrill, R. T., 1988a: Characteristics of the upper-tropospheric environmental flow around hurricanes. J. Atmos. Sci., 45, 16651677.

Merrill, R. T., 1988b: Environmental influences on hurricane intensification. J. Atmos. Sci., 45, 1678-1687.

Molinari, J., and D. Vollaro, 1989: External influences on hurricane intensity. Part I: Outflow layer eddy angular momentum fluxes. J. Atmos. Sci., 46, 1093-1104.

Molinari, J., and D. Vollaro, 1990: External influences on hurricane intensity. Part II: Vertical structure and response of the hurricane vortex. J. Atmos. Sci., 47, 1902-1918. 
Molinari, J., and D. Vollaro, 1993: Environmental controls on eye wall cycles and intensity change in Hurricane Allen (1980). Tropical Cyclone Disasters, J. Lighthill et al., Eds., Peking University Press, 328-337.

Molinari, J., D. Vollaro, and F. Robasky, 1992: Use of ECMWF operational analyses for studies of the tropical cyclone environment. Meteor. Atmos. Phys., 47, 127-144.

Molinari, J., S. Skubis, and D. Vollaro, 1995: External influences on hurricane intensity. Part III: Potential vorticity structure. $J$. Atmos. Sci., 52, 3593-3606.

Molinari, J., S. Skubis, D. Vollaro, F. Alsheimer, and H. E. Willoughby, 1998: Potential vorticity analysis of tropical cyclone intensification. J. Atmos. Sci., 55, 2632-2644.

Molinari, J., P. Dodge, D. Vollaro, and K. L. Corbosiero, 2006: Mesoscale aspects of the downshear reformation of a tropical cyclone. J. Atmos. Sci., 63, 341-354.

Montgomery, M. T., and R. K. Smith, 2014: Paradigms for tropical cyclone intensification. Australian Meteorological and Oceanographic Journal, 64, 37-66.

Pfeffer, R. L., and M. Challa, 1981: A numerical study of the role of eddy fluxes of momentum in the development of Atlantic hurricanes. J. Atmos. Sci., 38, 2393-2398.

Qian, Y. K., C. X. Liang, Q. Q. Liang, L. X. Lin, and Z. J. Yuan, 2011: On the forced tangentially-averaged radial-vertical circulation within vortices. Part II: The transformation of Tropical Storm Haima (2004). Adv. Atmos. Sci., 28, 1143-1158, doi: 10.1007/s00376-010-0060-x.

Rappin, E. D., M. C. Morgan, and G. J. Tripoli, 2011: The impact of outflow environment on tropical cyclone intensification and structure. J. Atmos. Sci., 68, 177-194.

Rodgers, E. B., S. W. Chang, J. Stout, J. Steranka, and J.-J. Shi, 1991: Satellite observations of variations in tropical cyclone convection caused by upper-tropospheric troughs. J. Appl. Meteor, 30, 1163-1184.

Rodgers, E. B., W. S. Olson, V. M. Karyampudi, and H. F. Pierce,
1998: Satellite-derived latent heating distribution and environmental influences in Hurricane Opal (1995). Mon. Wea. Rev., 126, 1229-1247.

Sawyer, J. S., 1956: The vertical circulation at meteorological fronts and its relation to frontogenesis. Proc. Roy. Soc. London, 234A, 346-362.

Shi, J. J., S. Chang, and S. Raman, 1997: Interaction between Hurricane Florence (1988) and an upper-tropospheric westerly trough. J. Atmos. Sci., 54, 1231-1247.

Smith, R. K., and M. T. Montgomery, 2015: Toward clarity on understanding tropical cyclone intensification. J. Atmos. Sci., 72, 3020-3031.

Sundqvist, H., 1970: Numerical simulation of the development of tropical cyclones with a ten-level model. Part I. Tellus, 22, 359-390.

Titley, D. W., and R. L. Elsberry, 2000: Large intensity changes in tropical cyclones: A case study of Supertyphoon Flo during TCM-90. Mon. Wea. Rev., 128, 3556-3573.

Willoughby, H. E., J. A. Clos, and M. G. Shoreibah, 1982: Concentric eye walls, secondary wind maxima, and the evolution of the hurricane vortex. J. Atmos. Sci., 39, 395-411.

Wu, C.-C., and H.-J. Cheng, 1999: An observational study of environmental influences on the intensity changes of Typhoons Flo (1990) and Gene (1990). Mon. Wea. Rev., 127, 3003 3031.

Wu, L. G., and B. Wang, 2000: A potential vorticity tendency diagnostic approach for tropical cyclone motion. Mon. Wea. Rev., 128, 1899-1911.

Yu, H., and H. J. Kwon, 2005: Effect of TC-trough interaction on the intensity change of two typhoons. Wea. Forecasting, 20, 199-211.

Zeng, Z. H., Y. Q. Wang, and C. -C. Wu, 2007: Environmental dynamical control of tropical cyclone intensity-An observational study. Mon. Wea. Rev., 135, 38-59. 